\title{
9. OXYGEN AND CARBON ISOTOPE STRATIGRAPHY AND MAGNETOSTRATIGRAPHY OF THE LAST 2.8 MA: PALEOCLIMATIC COMPARISONS BETWEEN THE NORWEGIAN SEA AND THE NORTH ATLANTIC ${ }^{1}$
}

\author{
E. Jansen, ${ }^{2}$ B. Slettemark, ${ }^{2}$ U. Bleil,${ }^{3}$ R. Henrich,${ }^{4}$ L. Kringstad, ${ }^{2}$ and S. Rolfsen ${ }^{2}$
}

\begin{abstract}
Continuous sediment sections spanning the last $2.8 \mathrm{Ma}$ have been studied using stable isotope stratigraphy and sedimentological methods. By using paleomagnetic reversals as a chronostratigraphic tool, climatic and paleoceanographic changes have been placed in a time framework. The results show that the major expansion of the Scandinavian Ice Sheet to the coastal areas occurred in the late Neogene period at about $2.8 \mathrm{Ma}$. Relatively high-amplitude glacials appeared until about $2 \mathrm{Ma}$. The period between 2.8 and $1.2 \mathrm{Ma}$ was marked by cold surface water conditions with only weak influx of temperate Atlantic water as compared with late Quaternary interglacials. During this period, climatic variations were smaller in amplitude than in the late Quaternary. The Norwegian Sea was a sink of deep water throughout the studied period but deep water ventilation was reduced and calcite dissolution was high compared with the Holocene. Deep water formed by other processes than today. Between 2 and $1.2 \mathrm{Ma}$, glaciations in Scandinavia were relatively small. A transition toward larger glacials took place during the period 1.2 to $0.6 \mathrm{Ma}$, corresponding with warmer interglacials and increasing influx of temperate surface water during interglacials. A strong thermal gradient was present between the Norwegian Sea and the northeastern Atlantic during the Matuyama (2.5-0.7 Ma). This is interpreted as a sign of a more zonal and less meridional climatic system over the region as compared with the present situation. The transition towards more meridionality took place over several hundred thousand yr. Only during the last $0.6 \mathrm{Ma}$ has the oceanographic and climatic system of the Norwegian Sea varied in the manner described from previous studies of the late Quaternary.
\end{abstract}

\section{INTRODUCTION}

The hydraulic piston coring on ODP Leg 104 provided, for the first time, continuous sections which extend beyond 400 k.y. in the Norwegian Sea (Eldholm, Thiede, Taylor, et al., 1987). These sections provide an opportunity to fill an important gap in the knowledge of the paleoclimatic evolution of high northern latitude regions in the Neogene and early Quaternary. In this paper we present paleoclimatic records for the last $2.8 \mathrm{Ma}$ with important climatic aspects for the area. The ODP drill sites are located close to the Scandinavian mainland and are thus proximal to the main accumulation area for the Scandinavian ice sheet. This location makes the sites ideal for the study of the waxing and waning of the ice sheet through time, and its effect on the neighboring oceanic environments. Continental records from the Scandinavian peninsula extend only to the last interglacial $(130 \mathrm{ka})$. New aspects of the younger geological evolution of northern Europe can be learned through the study of these sites. The variations in the oceanic circulation of the region are also one of the objectives of the study. The strong inflow of saline Atlantic surface waters into the Norwegian Sea presently has two major consequences:

1. It constitutes a strong heat flux that warms the European ;ub-arctic regions, making these regions climatically more agreesble than their counterparts on the western side of the Norwe,ian-Greenland Sea and North America.

\footnotetext{
${ }^{1}$ Eldholm, O., Thiede, J., Taylor, E., et al., 1989. Proc. ODP, Sci. Results, 04: College Station, TX (Ocean Drilling Program).

2 Department of Geology, Section B, Univ. of Bergen, Allegaten 41, N-5000 lergen, Norway.

3 Fachbereich Geowissenschaften, University of Bremen, Postfach 330440, 2800 Bremen, Federal Republic of Germany.

4 Geologisch-Paläontologisches Institut, University of Kiel, Olshausenstrasse 0, D-2300 Kiel, Federal Republic of Germany; now at GEOMAR, Forschungssntrum für Marine Geowissenschaften an der Universität Kiel, Wischholfstr. 1-3, -2300 Kiel, Federal Republic of Germany.
}

2 . It adds the necessary salt contribution which enables coldseason cooling of surface waters to produce large-scale vertical convection and deep-water formation. This process of deep-water formation provides the main ventilation of global deep waters in the modern ocean. Because this region exerts a global influence on deep water characteristics, it is important to better understand how this circulation regime was established, and how it has varied through time.

Because both the surface and deep-water environments of the Norwegian Sea and the North Atlantic are strongly related, we also include data from DSDP Site 610 from the northeastern North Atlantic to strengthen conclusions concerning environmental gradients that have existed in high latitudes during the last million yr.

\section{MATERIALS AND METHODS}

\section{Samples}

The location of the ODP and DSDP holes used in this study is shown in Figure 1. Parts of the sections utilized for this study were barren in foraminifers. We ascribe this phenomenon to two factors: (a) low foraminifer productivity, and (b) periods with extensive carbonate dissolution, especially a long dissolution period during Matuyama chron (Henrich, this volume). These factors decrease the stratigraphic resolution of the isotope records from the Norwegian Sea. Parts of the sediments in the deeper cores of Hole $644 \mathrm{~A}$ were partially disturbed by high natural gas pressures (Kvenvolden et al., this volume). Because of this disturbance, the sample recovery from these sections is more scattered than for the upper parts of the hole. Parts of the sediments belonging to the lower Matuyama Chron are probably disturbed by small slumps in Hole 643A. Elsewhere the paleomagnetic time control is reasonably good (Bleil, this volume, Clement and Robinson 1987).

At both Sites 642 and 644 , a double HPC-coring strategy was employed. Studies of the correlation of the offset holes is underway, but we are unable to present a spliced record by using data from offset holes at this time. The North Atlantic record comes from Site 610 which was drilled on the Feni Drift by DSDP Leg 94 in the northeasternmost North 


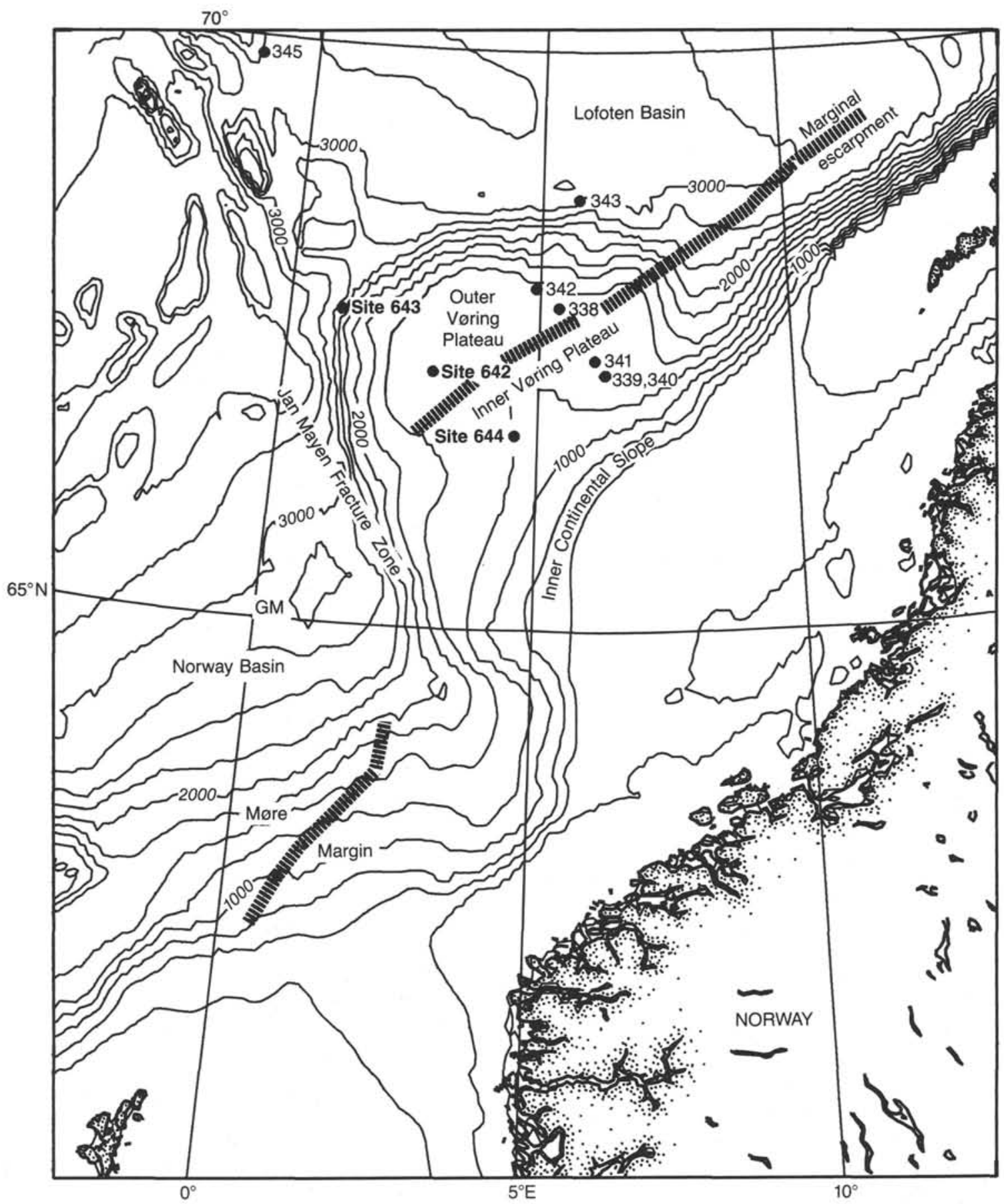

Figure 1. Bathymetry of the Norwegian continental margin, $60^{\circ}-70^{\circ} \mathrm{N}$ (contour interval, $250 \mathrm{~m}$ )

Atlantic (Fig. 1). Its location under the main flux of temperate North Atlantic waters flowing northwards into the Norwegian Sea and its good paleomagnetic record (Clement and Robinson 1987), makes it suitable for comparative study comprising a N-S transect at high northern latitudes. A pilot study has confirmed the potential for producing a detailed isotope record from Hole 610A (Jansen and Sejrup, 1987).

\section{Stable Isotopes}

The stable isotope measurements were made at the University of Bergen on a Finnigan MAT 251 mass spectrometer after reaction with orthophosphoric acid at $50^{\circ} \mathrm{C}$. The preparation line was an automatic on-line system with ten separate reaction chambers, following the design described by Shackleton et al. (1983) for a single-chambered manual version. Analytical precision of the system as defined by replicate measurements of carbonate standards is $\pm 0.06 \%$ for $\delta^{13} \mathrm{C}$ and \pm 0.07 for $\delta^{18} \mathrm{O}$. Measurements of $G$. bulloides in Hole $610 \mathrm{~A}$ were performed on the 250- to $1000-\mu \mathrm{m}$ fraction. All other specimens used in the isotope analysis were picked from the $125-$ to $1000-\mu \mathrm{m}$ fraction. The records from Leg 104 show a clear evolutionary trend within the $N$. pachyderma plexus with the first appearances of encrusted $N$. pachyderma close tc the Olduvai subchron at about 1.7 Ma (Spiegler and Jansen, this vol. ume). Thus, for the pre-Olduvai part, we analyzed reticulate left-coiling 
types of $N$. pachyderma sensu latd ( $N$. pseudopima aff. pachyderma of Spiegler and Jansen, this volume), whereas encrusted left-coiling $N$. pachyderma sensu stricto were used in the post-Olduvai part of the record.

The benthic isotope record from Hole 644A is based on the species Cassidulina teretis and Melonis barleeanum [also named Melonis zaandamae (Mackensen et al., 1985)] which are the only benthic species available in sufficient quantities stratigraphically to allow isotope analysis. Since the oxygen and carbon isotopic fractionation of these species is not well known in the literature, we performed an isotopic analysis of modern species to determine their isotopic disequilibria. We analyzed a number of samples from box-core tops in the Norwegian Sea to establish their isotopic relationship to the present water masses of the region (Fig. 2). Subsequently, we analyzed a number of paired analyses downhole to check the constancy of their isotopic offsets with time. The results suggest that $C$. teretis is close to oxygen isotopic equilibrium with ambient seawater, whereas $M$. barleeanum is consistently more negative by about $0.4 \%$ in $\delta{ }^{18} \mathrm{O}$, which agrees with results published previously for species of this genus (Graham et al., 1981; Woodruff et al., 1980). We consequently adjusted the measured values of $M$. barleeanum by adding $0.4 \%$ when producing the records on Figures 4,5 , and 7 , although we note some scatter in the downhole paired analyses (Fig. 3). This may have been caused by some downslope mixing or changes in the vital effects of these species.

\section{Paleomagnetism}

Procedures for magnetic measurements and demagnetization on the Leg 104 sediments are described by Bleil (this volume).

\section{Carbonate}

Carbonate percentages on Hole $610 \mathrm{~A}$ were measured in Bergen by means of a LECO-carbon analyzer. Carbonate measurements on the Leg 104 holes were performed in Kiel by an infrared gas analyzer utilizing photometric detection of $\mathrm{CO}_{2}$ after reacting the sample with phosphoric acid. The accuracy of the carbonate determinations is estimated to be within $10 \%$ of the measured value.

\section{Time Scale}

We have employed a time scale based on the identification of magnetic reversal boundaries (Bleil, this volume, Clement and Robinson, 1987). The boundaries picked are shown in Table 1. Outside of the reversal boundaries we have used linear interpolation by assuming constant sedimentation rates. The concept of constant sedimentation rates is obviously an oversimplification (see Henrich, this volume), since it is likely that the glacial-interglacial cycles produce large short term fluctuations in sedimentation rate. Thus the time scale employed is not able to date events outside of the magnetic fixed points with high accuracy. Yet it provides us with a first-order age estimate with an accuracy acceptable for the purpose of this study.

\section{RESULTS}

\section{Planktonic Oxygen Isotopes}

Figures 3 and 4 show the isotope results from Holes 644A and 643A plotted against depth below seafloor. We have proposed a first-order interpretation of isotope stages in Hole 643 based on the planktonic record, but the designation of isotope stages is difficult due to the discontinuity of the isotope stratigraphy and possible light-isotope excursions caused by low-salinity, glacial meltwater effects. We have marked the light-isotope peaks which correspond to high carbonate content. These can be considered the most pronounced interglacials, because warm periods of the Quaternary sediments in the Norwegian Sea and North Atlantic correspond with high biogenic carbonate content (Kellogg, 1976, Ruddiman et al., 1986a). However, there is not a straightforward correspondence between carbonate content and isotope values in Site 643. In fact, some intervals show-

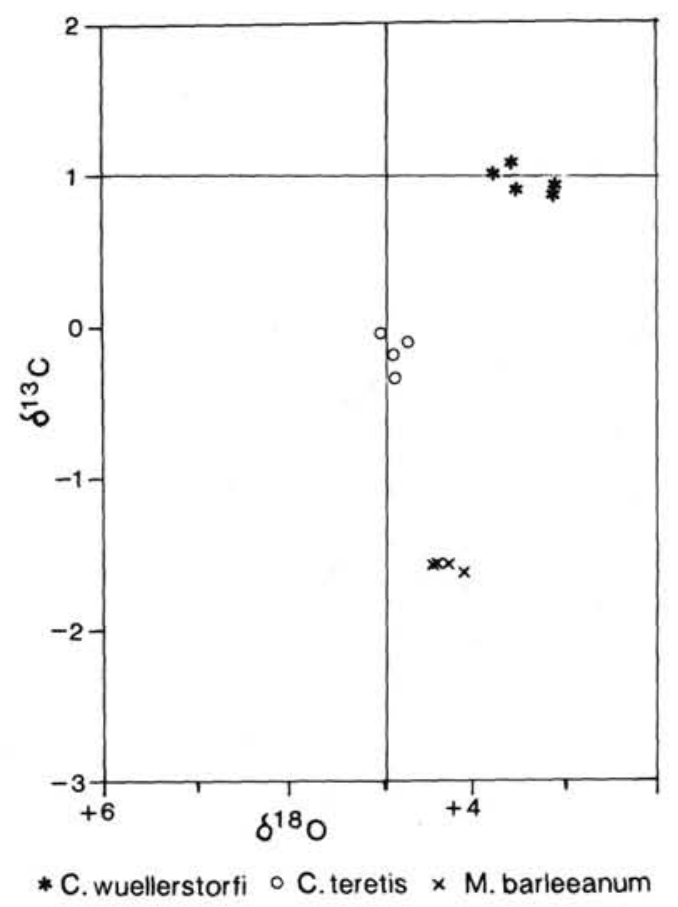

Figure 2. Stable isotope record of $C$. teretis, C. wuellerstorfi, and $M$. barleeanum in Holocene samples from the Norwegian Sea. Lines denote oxygen and carbon isotopic equilibrium.

Table 1. Mean oxygen and carbon isotope values and standard deviation during the Brunhes and Matuyama.

\begin{tabular}{|c|c|c|c|}
\hline Oxygen & $\bar{x}$ & $\sigma$ & $\mathrm{n}$ \\
\hline \multicolumn{4}{|l|}{$644 \mathrm{~A}$} \\
\hline \multicolumn{4}{|l|}{ Brunhes: } \\
\hline N. pachyderma sin. & 4.01 & 0.60 & 102 \\
\hline Benthics & 4.98 & 0.33 & 36 \\
\hline \multicolumn{4}{|l|}{ Matuyama: } \\
\hline N. pachyderma sin. & 2.69 & 0.77 & 84 \\
\hline Benthics & 4.66 & 0.41 & 201 \\
\hline \multicolumn{4}{|l|}{643} \\
\hline \multicolumn{4}{|l|}{ Brunhes: } \\
\hline N. pachyderma sin. & 4.09 & 0.62 & 99 \\
\hline $\begin{array}{l}\text { Matuyama: } \\
\text { N. pachyderma sin. }\end{array}$ & 3.40 & 0.57 & \\
\hline \multicolumn{4}{|l|}{ Carbon } \\
\hline \multicolumn{4}{|l|}{$644 A$} \\
\hline \multicolumn{4}{|l|}{ Brunhes: } \\
\hline $\begin{array}{l}\text { N. pachyderma } \sin \text {. } \\
\text { Matuyama: }\end{array}$ & -0.11 & 0.37 & 102 \\
\hline N. pachyderma sin. & -0.41 & 0.75 & 84 \\
\hline \multicolumn{4}{|l|}{643} \\
\hline \multicolumn{4}{|l|}{ Brunhes: } \\
\hline $\begin{array}{l}\text { N. pachyderma sin. } \\
\text { Matuyama: }\end{array}$ & 0.04 & 0.32 & 99 \\
\hline N. pachyderma sin. & -0.10 & 0.27 & 25 \\
\hline
\end{tabular}


$644 \mathrm{~A}$

Benthics
$644 \mathrm{~A}$

N. pachyderma (s)

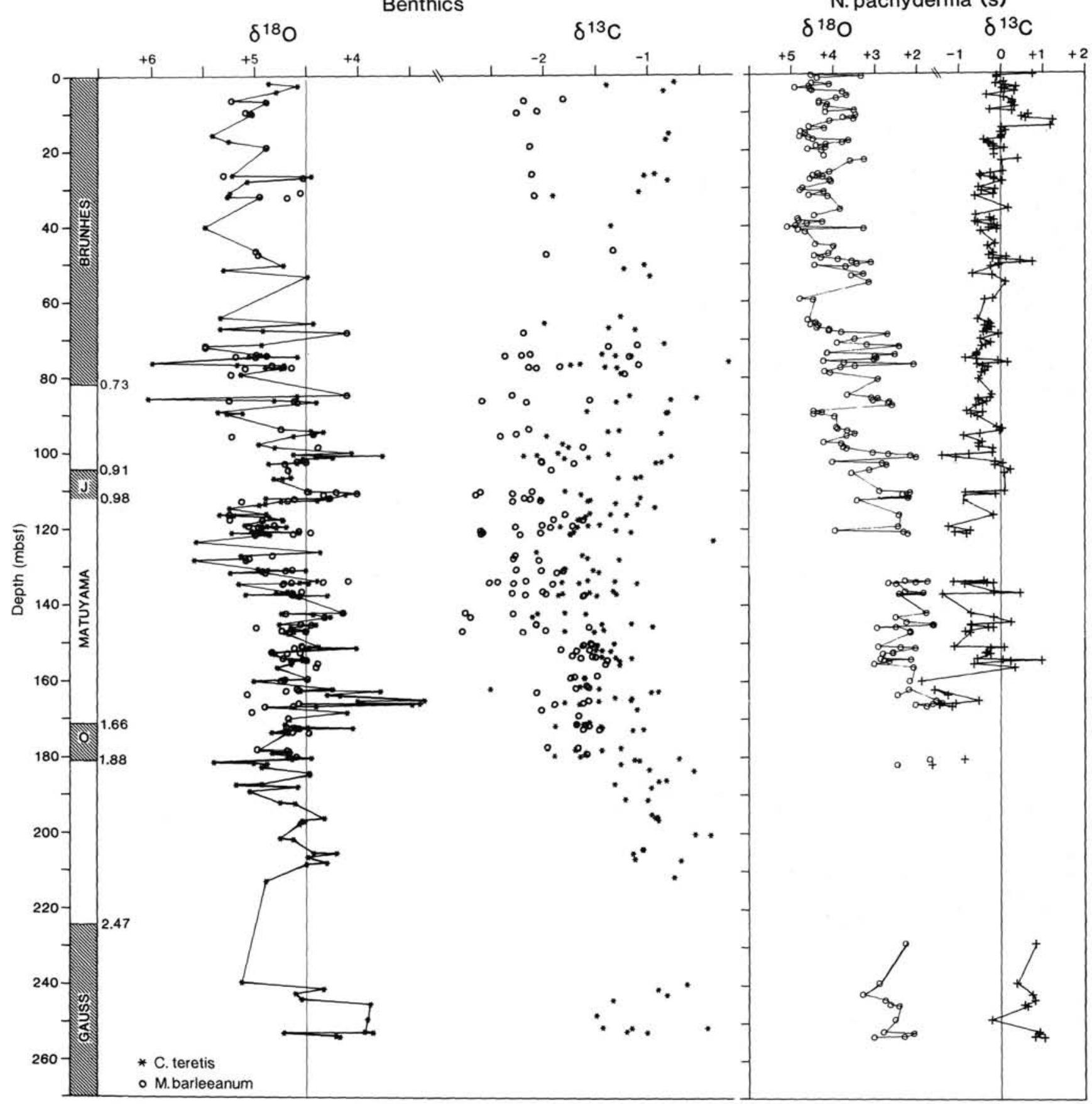

Figure 3. Stable isotope record and paleomagnetics of Hole 644A. The vertical line in the benthic oxygen isotope record refers to Holocene equilibrium values.

ing light planktonic $\delta^{18} \mathrm{O}$ values correspond with distinct glacial and deglacial lithologies (Henrich, this volume). The negative excursions in $\delta^{18} \mathrm{O}$ may indicate low salinity rather than true interglacials.

An extensive barren interval dominates the planktonic record from the uppermost Gauss to the Olduvai subchron. The average planktonic $\delta^{18} \mathrm{O}$ was clearly lighter during the Matuyama than in the Brunhes by $1.3 \%$ in Hole $644 \mathrm{~A}$ and $0.69 \% 0$ in Hole 643A (Tables 2 and 3). Starting at about 1.1 Ma and continuing until 0.6 Ma, the glacial $\delta^{18} \mathrm{O}$ levels become markedly heavier, and the interglacial peaks also became more positive in $\delta^{18} \mathrm{O}$.

\section{Benthic Oxygen Isotopes}

The availability of benthic foraminifers in Hole 644A (Table 4) is greater in the Matuyama than in the Brunhes. This contrasts with the abundance pattern of planktonic foraminifers which are most abundant in the Brunhes. Thus the benthic and 


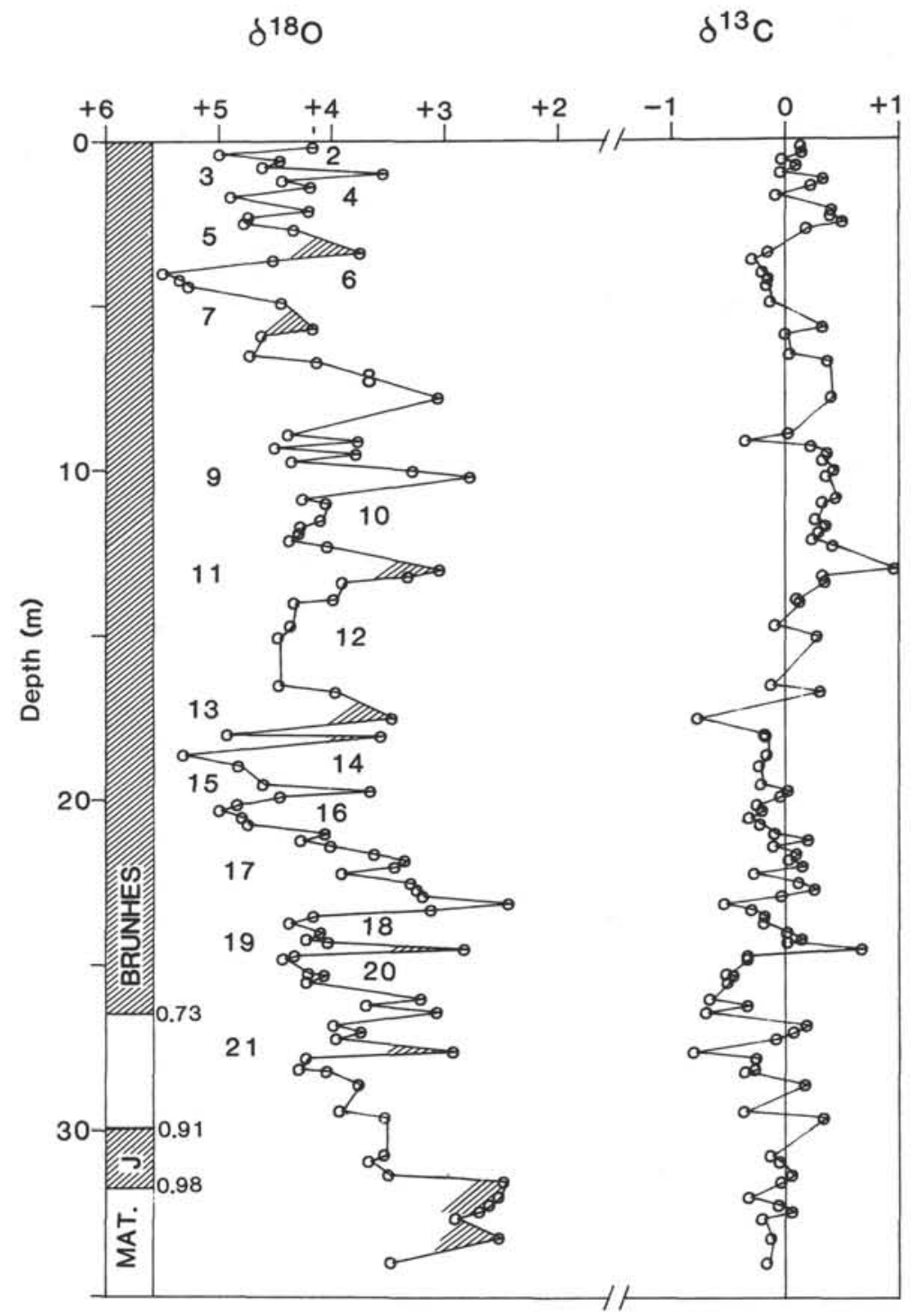

Figure 4. Stable isotope record and paleomagnetics of Hole 643A. Hached areas denote carbonate peaks.

planktonic records partly encompass different depth and age intervals, which make a direct comparison of the isotope curves difficult. The benthic $\delta^{18} \mathrm{O}$ record from Hole $644 \mathrm{~A}$ is also hampered by a barren interval in the uppermost Gauss and lower Matuyama, but this is less severe than the incomplete planktonic record. The benthic oxygen isotopic results from Hole 644A show distinct glacial excursions between 1.9 and $2.0 \mathrm{Ma}$, followed by a period with less climatic variation lasting to about 1.2 Ma, where heavier glacial excursions reappear in the record. Within the Olduvai is a period with markedly lighter $\delta^{18} \mathrm{O}$ values than seen in the rest of the Matuyama. The interglacial or light values of the Matuyama are generally at about the same level as, or lower than, the Holocene (Fig. 4). The noticeable transition from light to heavier values between 1.2 and $0.6 \mathrm{Ma}$ seen in the planktonic records does not show up in the benthic curve, which displays heavy values throughout the studied period (Figs. 3 and 4 and Table 2). This implies that there probably existed a strong oxygen isotopic gradient between surface and deep waters during the Matuyama, which diminished during the transition pe- riod. The gradient was on the average rather small during the last $0.6 \mathrm{Ma}$.

\section{Carbon Isotopes}

Planktonic $\delta^{13} \mathrm{C}$ values were heavy in the Gauss, whereas the average $\delta^{13} \mathrm{C}$ levels were lowest in the Matuyama, especially in Hole 644A (Table 1). The planktonic carbon isotope record shows a tendency toward heavier values within the Brunhes, where both the absolute values and the range of variation approaches that found in late Quaternary records of left-coiling $N$, pachyderma from the Norwegian Sea (Labeyrie and Duplessy, 1985, Beyer, 1988). In a general sense, the planktonic carbon isotopic values are lighter in the easternmost Hole 644A than in Hole 643A. A similar trend towards lighter $\delta^{13} \mathrm{C}$ values in the east is found in Holocene planktonic foraminifers in the region (Johannessen, 1987) and may represent an east-west gradient in the $\delta^{13} \mathrm{C}$ of near-surface waters. A similar gradient apparently existed from the inner to the outer Vøring Plateau throughout the 
Table 2. Isotope data from Hole 643A.

\begin{tabular}{|c|c|c|c|c|}
\hline $\begin{array}{l}\text { Depth } \\
\text { (mbsf) }\end{array}$ & $\begin{array}{l}\text { Core/sect } \\
104-643 \mathrm{~A} \text { - }\end{array}$ & Species & $\delta^{18} \mathrm{O}$ & $\delta^{13} \mathrm{C}$ \\
\hline 0.20 & $1-1,21 \mathrm{~cm}$ & pach. $s$ & .14 & 0.16 \\
\hline 0.40 & $1-1,40 \mathrm{~cm}$ & N. pach. $s$ & 97 & 0.17 \\
\hline 0.60 & $1-1,60 \mathrm{~cm}$ & N. pach. s & & -0.01 \\
\hline 0.80 & $1-1,81 \mathrm{~cm}$ & N. pach. $s$ & & 0.12 \\
\hline 1.01 & $1 \mathrm{~cm}$ & N. pach. $s$ & 3.52 & -0.02 \\
\hline 1.21 & $1-1,120 \mathrm{~cm}$ & N. pach. $s$ & 4.41 & 0.36 \\
\hline 1.41 & $1-1,138 \mathrm{~cm}$ & N. pach. $s$ & 4.16 & 0.25 \\
\hline 1.70 & $1-2,40 \mathrm{~cm}$ & N. pach. $s$ & 4.87 & -0.06 \\
\hline 2.14 & $1-2,64 \mathrm{~cm}$ & N. pach. $s$ & 4.17 & 0.43 \\
\hline 2.31 & $1-2,81 \mathrm{~cm}$ & N. pach. $s$ & 4.71 & 0.42 \\
\hline 2.51 & $1-2,100 \mathrm{~cm}$ & N. pach. $s$ & 4.75 & 0.53 \\
\hline 2.71 & $1-2,120 \mathrm{~cm}$ & N. pach. s & & 0.21 \\
\hline 3.40 & $1-3,40 \mathrm{~cm}$ & N. pach. $s$ & 3.72 & -0.13 \\
\hline 3.62 & $1-3,62 \mathrm{~cm}$ & N. pach. s & 4.49 & -0.27 \\
\hline 4.01 & $1-3,101 \mathrm{~cm}$ & N. pach. s & & -0.18 \\
\hline 4.21 & 1-3, & N. po & & \\
\hline 4.41 & $1-3$, & N. $p a$ & 4 & -0.14 \\
\hline 4.91 & $1-4$, & N. pach. $s$ & 4.42 & -0.11 \\
\hline 5.70 & $2-1,40 \mathrm{~cm}$ & N. pach. $s$ & 4.14 & 0.35 \\
\hline 5.90 & $2-1,60 \mathrm{~cm}$ & N. pach. $s$ & 4. & 0.02 \\
\hline 6.50 & $2-1,120 \mathrm{~cm}$ & N. pach. $s$ & 4.70 & 0.06 \\
\hline 6.72 & $2-1,1$ & N. pach. $s$ & & 0.40 \\
\hline 7.80 & $2-2,1$ & N. pach. $s$ & & 0.43 \\
\hline 8.90 & $2-3,6$ & $N . P$ & & 0.05 \\
\hline 9.12 & $2-3,82 \mathrm{~cm}$ & N. pach. $s$ & 3.74 & -0.33 \\
\hline 9.30 & $2-3,100 \mathrm{~cm}$ & N. pach. s & 4.48 & 0.25 \\
\hline 9.50 & $2-3,120$ & N. $p a$ & & 0.39 \\
\hline 9.72 & $2-3$ & N. p & & 0.35 \\
\hline 10.01 & $2-4,2$ & N. $p$ & & 0.45 \\
\hline 10.20 & $2-4,4$ & N. pach. $s$ & & 0.38 \\
\hline 10.86 & $2-4,1$ & N. $p$ & & 0.47 \\
\hline 11.00 & $2-4,1$ & N. $p$ & 4. & 0.35 \\
\hline 11.51 & $2-5,21 \mathrm{~cm}$ & N. pach. $s$ & 4. & 0.29 \\
\hline 11.70 & $2-5,40 \mathrm{~cm}$ & N. pach. $s$ & 4.25 & 0.38 \\
\hline 11.90 & $2-5,60 \mathrm{~cm}$ & N. pach. $s$ & & 0.32 \\
\hline 12.12 & $2-5,80 \mathrm{~cm}$ & N. pach. $s$ & & 0.26 \\
\hline 12.30 & $2-5,100 \mathrm{~cm}$ & N. pach. s & 4.01 & 0.44 \\
\hline 13.01 & $2-6,21 \mathrm{~cm}$ & N. $p c$ & 2 & 0.98 \\
\hline 13.20 & $2-6$, & N. $p$ & 0 & 0.35 \\
\hline 13.40 & $2-6$, & N. $P$ & 3 & 0.37 \\
\hline 13.91 & $2-6$, & N. $p c$ & 3 & 0.12 \\
\hline 14.00 & $2-6,1$ & N. pach. $s$ & 4.31 & 0.15 \\
\hline 14.70 & $2-7,40 \mathrm{~cm}$ & N. pach. s & 4.34 & -0.07 \\
\hline 15.05 & $3-1,25 \mathrm{~cm}$ & N. pach. s & 4.45 & 0.30 \\
\hline 16.50 & $3-2,20 \mathrm{~cm}$ & N. pach. $s$ & 4.44 & -0.10 \\
\hline 16.72 & $3-2,42 \mathrm{~cm}$ & N. pach. $s$ & 3.94 & 0.33 \\
\hline 17.50 & $3-2,120 \mathrm{~cm}$ & N. pach. s & 3.44 & \\
\hline 18.00 & $3-3,20 \mathrm{~cm}$ & N. pach. $s$ & 4.90 & -0 \\
\hline 18.05 & $3-3,2$ & N. pach. s & 4 & \\
\hline 18.62 & $3-3,8$ & N. $F$ & 9 & \\
\hline 18. & $\mathrm{~m}$ & N. pc & 0 & 21 \\
\hline 19.50 & $3-4,2$ & N. pach. $s$ & 4. & -0.19 \\
\hline 19.72 & $3-4,42 \mathrm{~cm}$ & N. pach. $s$ & 3.63 & 0.05 \\
\hline 19.88 & $3-4,58 \mathrm{~cm}$ & N. pach. s & 4.43 & -0.02 \\
\hline 20.12 & $3-4,82 \mathrm{~cm}$ & N. pach. $s$ & 4.81 & -0.23 \\
\hline 20.30 & $-4,100 \mathrm{~cm}$ & N. pach. s & 4.96 & -0.18 \\
\hline 20.52 & $3-4,122 \mathrm{~cm}$ & N. pach. $s$ & 4.76 & $-c$ \\
\hline 20.72 & $3-4$, & N. pach. $s$ & 2 & \\
\hline 21.00 & $3-5,2$ & N. pach. $s$ & 4.0 & -0.07 \\
\hline 21.20 & 5,4 & N. pach. $s$ & 4. & 0.23 \\
\hline 21.38 & -5, & N. pach. $s$ & 3.98 & -0.08 \\
\hline 21.62 & 5 & N. pach. $s$ & 0 & 0.12 \\
\hline 21.80 & $\mathrm{~cm}$ & N. pach. $s$ & 3.32 & 0.05 \\
\hline 22.00 & $3-5,111 \mathrm{~cm}$ & N. pach. $s$ & 3.42 & 0.17 \\
\hline 22.20 & $3-5,140 \mathrm{~cm}$ & N. pach. s & 3.89 & -0.26 \\
\hline 22.5 & $3-6,20 \mathrm{~cm}$ & N. pach. s & 3.28 & 0.14 \\
\hline 22.7 & $-6,40 c$ & N. pach. s & 3.22 & 0.28 \\
\hline 22.8 & $3-6,58$ & N. pach. $s$ & 3.17 & -0.01 \\
\hline 23.1 & $\mathrm{n}$ & N. $p$ & 2.41 & -0.52 \\
\hline 23.30 & 0, rov cim & N. pach. $s$ & 3.10 & \\
\hline 23.50 & $3-6,120 \mathrm{~cm}$ & N. pach. $s$ & 4.14 & -0.16 \\
\hline 23.70 & $3-6,140 \mathrm{~cm}$ & N. pach. s & 4.35 & -0.17 \\
\hline 24.00 & $3-7,25 \mathrm{~cm}$ & N. pach. $s$ & 4.07 & 0.04 \\
\hline 24.20 & $3-7,40 \mathrm{~cm}$ & N. pach. $s$ & 4.20 & 0.17 \\
\hline 24.30 & $3-7 ., 55 \mathrm{~cm}$ & N. pach. $s$ & 4.00 & 0.05 \\
\hline 24.50 & $4-1,20 \mathrm{c}$ & N. pach. s & 2.80 & 0.70 \\
\hline 24.70 & $4-1,40 \mathrm{~cm}$ & N. pach. $s$ & 4.30 & -0.31 \\
\hline
\end{tabular}

Table 2 (continued).

\begin{tabular}{|c|c|c|c|c|}
\hline $\begin{array}{l}\text { Depth } \\
\text { (mbsf) }\end{array}$ & $\begin{array}{l}\text { Core/sect } \\
104-643 \mathrm{~A}-\end{array}$ & Species & $\delta^{18} \mathrm{O}$ & $\delta^{13} \mathrm{C}$ \\
\hline 24.80 & $4-1,80 \mathrm{~cm}$ & N. pach, s & 4.40 & -0.31 \\
\hline 25.24 & $4-1,94 \mathrm{~cm}$ & N. pach. $s$ & 4.18 & -0.50 \\
\hline 25.30 & $4-1,100 \mathrm{~cm}$ & N. pach. s & 4.04 & -0.44 \\
\hline 25.50 & $4-1,120 \mathrm{~cm}$ & N. pach. s & 4.19 & -0.49 \\
\hline 26.00 & $4-2,20 \mathrm{~cm}$ & N. pach. $s$ & 3.19 & -0.64 \\
\hline 26.20 & $4-2,40 \mathrm{~cm}$ & N. pach. $s$ & 3.67 & -0.31 \\
\hline 26.40 & $4-2,60 \mathrm{~cm}$ & N. pach. $s$ & 3.04 & -0.68 \\
\hline 26.80 & $4-1,100 \mathrm{~cm}$ & N. pach. $s$ & 3.96 & 0.21 \\
\hline 27.00 & $4-2,120 \mathrm{~cm}$ & N. pach. $s$ & 3.72 & 0.10 \\
\hline 27.20 & $4-2,140 \mathrm{~cm}$ & N. pach. s & 3.94 & -0.06 \\
\hline 27.60 & $4-3,40 \mathrm{~cm}$ & N. pach. $s$ & 2.90 & -0.79 \\
\hline 27.80 & $4-3,60 \mathrm{~cm}$ & N. pach. s & 4.20 & -0.23 \\
\hline 28.14 & $4-3,92 \mathrm{~cm}$ & N. pach. $s$ & 4.27 & -0.25 \\
\hline 28.20 & $4-3,100 \mathrm{~cm}$ & N. pach. s & 4.02 & -0.34 \\
\hline 28.60 & $4-3,1$ & N. pach. $s$ & 3.73 & 0.20 \\
\hline 29.40 & $4-4,6$ & N. pach. $s$ & 3.90 & -0.34 \\
\hline 29.60 & $4-4,8$ & N. pach. $s$ & 3.50 & 0.36 \\
\hline 30.70 & $4-5,4$ & N. pach. s & 3.51 & -0.11 \\
\hline 30.90 & $4-5,6$ & N. pach. $s$ & 3.65 & -0.03 \\
\hline 31.30 & $4-5$, & N. pach. $s$ & 3.47 & 0.08 \\
\hline 31.50 & $4-5,1$ & N. pach. $s$ & 2.45 & -0.01 \\
\hline 31.96 & $4-6,16 \mathrm{~cm}$ & N. pach. $s$ & 2.50 & -0.30 \\
\hline 32.20 & $4-6,40 \mathrm{~cm}$ & N. pach. $s$ & 2.58 & -0.04 \\
\hline 32.40 & $4-6,60$ & N. pach. s & 2.67 & 0.08 \\
\hline 32.60 & $4-6,80$ & N. pach. $s$ & 2.88 & -0.19 \\
\hline 33.20 & $4-6,140$ & N. pach. $s$ & 2.49 & -0.10 \\
\hline 33.94 & $5-1,25 \mathrm{c}$ & N. pach. $s$ & 3.45 & -0.14 \\
\hline 43.50 & $6-1,8$ & N. pach. $s$ & 3.45 & 0.17 \\
\hline 45.22 & $6-2$, & N. pach. $s$ & 3.60 & -1.58 \\
\hline 51.26 & $6-6,4$ & N. pach. $s$ & 2.75 & -1.43 \\
\hline 55.13 & $7-2,82 \mathrm{~cm}$ & N. pach. $s$ & 3.24 & 0.51 \\
\hline 55.35 & $7-2,105 \mathrm{~cm}$ & N. pach. $s$ & 3.15 & 0.66 \\
\hline 55.72 & $7-2,142 \mathrm{~cm}$ & N. pach. s & 1.93 & -0.15 \\
\hline 56.42 & $7-3,62 \mathrm{~cm}$ & N. pach. $s$ & 3.22 & 0.82 \\
\hline 57.02 & $7-3,122 \mathrm{~cm}$ & N. pach. $s$ & 3.07 & 0.73 \\
\hline 57.22 & $7-3,142 c$ & N. pach. $s$ & 3.29 & 0.80 \\
\hline 57.51 & $7-4,21 \mathrm{~cm}$ & N. pach. $s$ & 3.51 & 1.14 \\
\hline 57.72 & $7-4,41$ & N. pach. $s$ & 3.74 & 1.11 \\
\hline 58.53 & $7-4,122 \mathrm{~cm}$ & N. pach. $s$ & 1.45 & -1.03 \\
\hline 58.72 & $7-4,142 \mathrm{~cm}$ & N. pach. $s$ & 4.54 & 1.66 \\
\hline
\end{tabular}

studied period and marks a general feature of the surface circulation and/or productivity.

In the upper Gauss and the pre-Olduvai parts of the Matuyama the benthic $\delta^{13} \mathrm{C}$ values appear, on the average, heavier than those of the rest of the Matuyama younger than about $1.8 \mathrm{Ma}$. A decrease in $\delta^{13} \mathrm{C}$ is indicated at about $1.8 \mathrm{Ma}$.

\section{North Atlantic Isotope Record}

Figure 5 shows the planktonic isotope record of $G$. bulloides from Hole $610 \mathrm{~A}$ as a function of estimated age, based on the paleomagnetic time scale. Also shown is the benthic isotope record of Shackleton et al. (1984) from DSDP Hole 552A on the same time scale, based on the magnetic reversal boundaries tabulated by Shackleton and Hall (1984). This hole was drilled on the flank of the Hatton Rockall Plateau (Fig. 1), at about the same water depth as Site 610 . Because the paucity of benthic foraminifers in Hole 610A makes it impossible to produce a record with the same resolution as the planktonic, we have chosen to use the record from nearby Site 552 as representative of isotope variations in this part of the deep North Atlantic. The simple time scale used makes detailed correlations between the two sites problematic. However, it should be possible to make general comparisons of the isotopic fluctuations. In contrast to the Norwegian Sea records, there is no obvious reduction in benthic-to-planktonic oxygen isotopic gradients in the upper Matuyama-lower Brunhes from the North Atlantic records (Site 610 G. bulloides vs. Site 552 benthic) (Fig. 5). The benthic record of 
Table 3. Planktonic foraminiferal isotope data from Hole 644A.

\begin{tabular}{|c|c|c|c|c|}
\hline $\begin{array}{l}\text { Depth } \\
\text { (mbsf) }\end{array}$ & $\begin{array}{l}\text { Core/sect } \\
104-644 \mathrm{~A}-\end{array}$ & Species & $\delta^{18} \mathrm{O}$ & $\delta^{13} \mathrm{C}$ \\
\hline 0.22 & $\mathrm{~cm}$ & oach. $s$ & 8 & 0.77 \\
\hline 0.59 & & N. pach. $s$ & & -0.09 \\
\hline 1.02 & $1-1,102 \mathrm{~cm}$ & N. pach. $s$ & & \\
\hline 2.30 & $1-2,82 \mathrm{~cm}$ & N. pach. $s$ & 4.48 & 0.08 \\
\hline 2.72 & $1-2,122 \mathrm{~cm}$ & N. pach. $s$ & 4.06 & -0.11 \\
\hline 3.22 & $1-3,22 \mathrm{~cm}$ & N. pach. $s$ & 4.54 & 0.13 \\
\hline 3.59 & $1-3,59 \mathrm{~cm}$ & N. pach. $s$ & 4.88 & 0.38 \\
\hline 4.02 & $1-3,102 \mathrm{~cm}$ & N. pach. $s$ & 4.52 & 0.04 \\
\hline 4.22 & $1-3,122 \mathrm{~cm}$ & N. pach. $s$ & 4.47 & 0.10 \\
\hline 4.72 & $1-4,42 \mathrm{~cm}$ & N. pach. $s$ & 3.74 & 0.33 \\
\hline 5.72 & $1-4,122 \mathrm{~cm}$ & N. pach. $s$ & 3.64 & -0.33 \\
\hline 6.42 & $1-5,42 \mathrm{~cm}$ & N. pach. $s$ & 3.89 & 0.08 \\
\hline 7.22 & $1-5,122 \mathrm{~cm}$ & N. pach. $s$ & 4.29 & 0.29 \\
\hline 7.72 & $1-6,22 \mathrm{~cm}$ & N. pach. $s$ & 4.30 & 0.29 \\
\hline 8.09 & $1-6,59 \mathrm{~cm}$ & N. pach. $s$ & & 0.27 \\
\hline 8.72 & $1-6,1$ & N. pach. $s$ & & 0.34 \\
\hline 9.62 & $2-1,4$ & N. pach. $s$ & & \\
\hline 10.00 & $2-1$, & N. pach. $s$ & & 0.28 \\
\hline 10.92 & $2-2$ & N. & & 0.67 \\
\hline 11.52 & $2-2$ & N. pach. $s$ & & 0.50 \\
\hline 11.90 & $2-2,122 \mathrm{~cm}$ & N. pach. $s$ & 7 & 0.60 \\
\hline 12.42 & $2-3,62 \mathrm{~cm}$ & N. pach. $s$ & 4.05 & 1.25 \\
\hline 13.92 & $2-4,22 \mathrm{~cm}$ & N. pach. $s$ & 4.54 & 1.20 \\
\hline 14.32 & $2-4$, & N. pach. $s$ & 4.17 & 0.04 \\
\hline 15.12 & $2-4$, & N. pach. $s$ & 4.74 & 0.12 \\
\hline 15.62 & $2-5$, & N. pach. $s$ & 4.63 & 0.00 \\
\hline 16.42 & $3-1,22 \mathrm{~cm}$ & N. pach. $s$ & 4.76 & 0.04 \\
\hline 16.82 & $3-1,6$ & N. pach. $s$ & 4.55 & 0.02 \\
\hline 17.22 & $3-1$ & N. pach. $s$ & 4.44 & 0.02 \\
\hline 17.60 & $3-1$, & N. pach. $s$ & 3.60 & -0.39 \\
\hline 18.12 & $3-2,42 \mathrm{~cm}$ & N. pach. $s$ & 3.74 & -0.30 \\
\hline 18.52 & $3-2,82 \mathrm{~cm}$ & N. pach. $s$ & 4.14 & -0.28 \\
\hline 18.90 & $3-2,122 \mathrm{~cm}$ & N. pach. $s$ & 4.37 & -0.17 \\
\hline 19.42 & $3-3,22 \mathrm{~cm}$ & N. pach. s & 4 & -0.16 \\
\hline 19.82 & $3-3,62 \mathrm{~cm}$ & N. pach. $s$ & & 0.09 \\
\hline 20.20 & $3-3$, & N. pach. $s$ & & - \\
\hline 21. & $3-4$, & N. & & 15 \\
\hline 22 & $3-$ & $s$ & & 42 \\
\hline 23.02 & 3-5, & $s$ & 6 & 02 \\
\hline 25.92 & $4-1$, & N. pach. $s$ & 5 & 0.06 \\
\hline 26.32 & $4-1,62 \mathrm{~cm}$ & N. pach. $s$ & 4.32 & -0.24 \\
\hline 26.72 & $4-1,102 \mathrm{~cm}$ & N. pach. $s$ & 4.45 & -0.50 \\
\hline 27.12 & $4-1,142 \mathrm{~cm}$ & N. pach. $s$ & 4.20 & -0.46 \\
\hline 27.62 & $4-2,42 \mathrm{~cm}$ & N. pach. $s$ & 4.51 & -0.16 \\
\hline 28.02 & $4-2,82 \mathrm{~cm}$ & N. pach. $s$ & 4.04 & -0.15 \\
\hline 28.40 & $4-2,122 \mathrm{~cm}$ & N. pach. $s$ & 4.00 & 0.05 \\
\hline 30.12 & $4-3,142 \mathrm{~cm}$ & N. pach. $s$ & 4.69 & -0.52 \\
\hline 30.62 & $4-4,42 \mathrm{~cm}$ & N. pach. $s$ & 4.74 & -0.13 \\
\hline 31.02 & $4-4,82 \mathrm{~cm}$ & N. pach. $s$ & 4.18 & -0.45 \\
\hline 31.92 & $4-5,22 \mathrm{~cm}$ & N. pach. $s$ & 4.54 & -0.17 \\
\hline 32.32 & $4-5,62 \mathrm{~cm}$ & N. pach. $s$ & 4.09 & -0.61 \\
\hline 35.6 & $5-1,42$ & N. pach. $s$ & 3 & 0.19 \\
\hline 37. & & N. $p$ & 4 & $-\mathrm{C}$ \\
\hline 38 & & $N$ & & - \\
\hline 38 & & $N$ & 8 & \\
\hline 39.02 & & & 1 & \\
\hline 39.40 & $5-3,1$ & pach. $s$ & & \\
\hline 39.92 & $5-4,2$ & N. pach. $s$ & & - \\
\hline 40.32 & $5-4,62 \mathrm{~cm}$ & N. pach. $s$ & 5.06 & - \\
\hline 40.72 & $5-4,102 \mathrm{~cm}$ & N. pach. $s$ & 3.23 & -0.27 \\
\hline 41.07 & $5-4,137 \mathrm{~cm}$ & N. pach. $s$ & 4.79 & -0.08 \\
\hline 41.62 & $5-5,42 \mathrm{~cm}$ & N. pach. $s$ & 4.62 & -0.47 \\
\hline 44.92 & $6-1,22 \mathrm{~cm}$ & N. pach. $s$ & 4.38 & -0.13 \\
\hline 45.48 & $6-2,78 \mathrm{~cm}$ & N. pach. $s$ & 3.95 & -0.31 \\
\hline 47.40 & $6-2,122 \mathrm{~cm}$ & N. pach. $s$ & 4.07 & -0.17 \\
\hline 47.92 & $6-3,22 \mathrm{~cm}$ & N. pach. $s$ & 4.41 & -0.27 \\
\hline 48.48 & $6-3,78 \mathrm{~cm}$ & N. pach. $s$ & 4.24 & 0.15 \\
\hline 48.90 & $6-3,122 \mathrm{~cm}$ & N. pach. $s$ & 3.84 & -0.18 \\
\hline 49.42 & $6-4,22 \mathrm{~cm}$ & N. pach. s & 3.51 & 0.48 \\
\hline 49.79 & $6-4,59 \mathrm{~cm}$ & N. pach. $s$ & 3.05 & 0.77 \\
\hline 50.21 & $6-4,100 \mathrm{~cm}$ & N. pach. $s$ & 3.39 & -0.04 \\
\hline 50.92 & $6-5,22 \mathrm{~cm}$ & N. pach. $s$ & 3.66 & -0.23 \\
\hline 50.48 & $6-5,79 \mathrm{~cm}$ & N. pach. $s$ & 4.40 & -0.04 \\
\hline 52.79 & $6-6,59 \mathrm{~cm}$ & N. pach. $s$ & 3.23 & -0.66 \\
\hline 53.21 & $6-6,101 \mathrm{~cm}$ & N. pach. $s$ & 3.52 & -0.19 \\
\hline 55.03 & $7-1,84 \mathrm{~cm}$ & N. pach. $s$ & 3.11 & 0.12 \\
\hline 59.35 & $7-4,65 \mathrm{~cm}$ & N. pach. $s$ & 4.75 & -0.18 \\
\hline
\end{tabular}

Table 3 (continued).

\begin{tabular}{|c|c|c|c|c|}
\hline $\begin{array}{l}\text { Depth } \\
\text { (mbsf) }\end{array}$ & $\begin{array}{l}\text { Core/sect } \\
104-644 \mathrm{~A}-\end{array}$ & Species & $\delta^{18} \mathrm{O}$ & $\delta^{13} \mathrm{C}$ \\
\hline 59.56 & s & pach. $s$ & 43 & 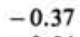 \\
\hline & & Vach. $s$ & 56 & (2) \\
\hline 65.60 & & & 37 & \\
\hline 600 & . & pach. $s$ & 50 & 0.24 \\
\hline 6.40 & & N. pach. $s$ & 4.30 & \\
\hline 66.90 & , & N. pach. $s$ & 34 & -0.21 \\
\hline 67.31 & & N. pach. $s$ & 4.04 & -0.32 \\
\hline 67.72 & 102 & N. pach. $s$ & 06 & -0.33 \\
\hline 68.10 & & N. pach. $s$ & & \\
\hline 68.62 & 4 & N. pach. $s$ & 66 & \\
\hline 69.92 & & N. pach. s & & \\
\hline 70. & 5, & N. $P$ & & \\
\hline 71.42 & & & & -0.35 \\
\hline 71.8 & 6,6 & N. P & & \\
\hline & & $N$ noc & & -0.56 \\
\hline 74.0 & 9-1, & & 48 & -0.57 \\
\hline 74.4 & & N. pach. $s$ & & \\
\hline 74.9 & & ach. $s$ & & \\
\hline 75.2 & & N. pach. $s$ & & \\
\hline & & 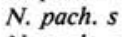 & & -0.05 \\
\hline 76.1 & 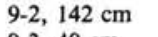 & N. pach. $s$ & & 18 \\
\hline 76.6 & & ch. $s$ & 03 & \\
\hline 77.02 & r & N. $p$ & & \\
\hline 77.43 & & N. pach. s & & \\
\hline 78.43 & $9-4$, & N. & & \\
\hline 78.7 & & & & \\
\hline 80.52 & & & & \\
\hline & & $n$ & & -0.20 \\
\hline 85.4 & & & & \\
\hline 85.6 & & N. pach. $s$ & 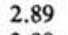 & $-c$ \\
\hline 86.1 & & N.. & & \\
\hline 86.4 & & $N . p$ & & $-c$ \\
\hline 86 & & $N$. & & \\
\hline 87.4 & & N. pach. $s$ & & -0.58 \\
\hline 88.9 & & ch. $s$ & & \\
\hline 89.2 & $\mathrm{~m}$ & N. pach. $s$ & & -0.41 \\
\hline 89.7 & & & & \\
\hline 90. & & N. $F$ & & $-c$ \\
\hline & & & & \\
\hline 93. & & N. $p$ & & .04 \\
\hline & & & & \\
\hline 94.5 & & N. $p$ & & 48 \\
\hline & & & & \\
\hline 97. & & N. $F$ & & -0.4 \\
\hline & & $s$ & & \\
\hline 98. & & $s$ & & 5 \\
\hline & & $s$ & & \\
\hline & & 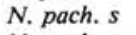 & & \\
\hline & 1 &.$t$. & & -0.7 \\
\hline & & & & \\
\hline & & $N$ & & -1.0 \\
\hline & & & & \\
\hline & & $s$ & & 0 \\
\hline & & & & \\
\hline & & $N$. & & 24 \\
\hline & & & & \\
\hline & & 1. & & \\
\hline & & & & \\
\hline & & $N$. & & \\
\hline & & & & \\
\hline & & & & .81 \\
\hline & & & & \\
\hline & & & & \\
\hline & & & & \\
\hline & & & & \\
\hline & & & & \\
\hline & & & & \\
\hline & & & & \\
\hline & & & & \\
\hline & & & & \\
\hline & & & & \\
\hline & & $N$. & & \\
\hline & & & & \\
\hline & & N. & & -1. \\
\hline & & & & \\
\hline & & & & \\
\hline & & & & \\
\hline
\end{tabular}


Table 3 (continued).

\begin{tabular}{|c|c|c|c|c|}
\hline $\begin{array}{l}\text { Depth } \\
\text { (mbsf) }\end{array}$ & $\begin{array}{l}\text { Core/sect } \\
104-644 \mathrm{~A}-\end{array}$ & Species & $\delta^{18} \mathrm{O}$ & $\delta^{13} \mathrm{C}$ \\
\hline 4.43 & $16-4,23 \mathrm{~cm}$ & N. pach. $s$ & 2.21 & 0.26 \\
\hline 55.02 & 16-4, & N. P & & 0.71 \\
\hline 145.20 & $16-4,120 \mathrm{~cm}$ & N. pach. $s$ & 1.55 & -0.68 \\
\hline 149.93 & $16-5,22 \mathrm{~cm}$ & N. pach. $s$ & 2.91 & -0.17 \\
\hline 145.72 & $16-5,102 \mathrm{~cm}$ & N. pach. $s$ & 2.45 & -0.29 \\
\hline 146.92 & $16-5,120 \mathrm{~cm}$ & N. pach. s & 2.12 & -0.83 \\
\hline 147.12 & $16-5,142 \mathrm{~cm}$ & N. pach. $s$ & 2.11 & -0.71 \\
\hline 150.92 & $17-2,22 \mathrm{~cm}$ & N. pach. s & 2.88 & -1.10 \\
\hline 151.12 & $17-$ & N. & & 0.10 \\
\hline 151.32 & $17-2,6$ & N. pach. $s$ & & -0.23 \\
\hline 152.42 & 17. & $N$ & & 32 \\
\hline 152.62 & $17-3,42$ & N. pach. $s$ & & -0.35 \\
\hline 152.82 & $17-3,62$ & N. pach. s & & -0.23 \\
\hline 154.12 & $17-4,42$ & N. pach.s & 2. & -0.55 \\
\hline 154.32 & $17-4,102$ & N. pach.s & 2. & 0.06 \\
\hline 154.52 & $17-4,1$ & N. pach. s & 2. & 0.98 \\
\hline 154.72 & $17-4,142$ & N. pach. s & 2. & 0.25 \\
\hline 155.42 & $17-5,22 \mathrm{~cm}$ & N. pach. $s$ & 2.98 & -0.63 \\
\hline 156.42 & $17-5,122 \mathrm{~cm}$ & N. $p$ & 2. & 0.36 \\
\hline 159.92 & $18-1,1$ & N. pach. $s$ & 2. & -1.86 \\
\hline 163.62 & $18-4,4$ & N. pach. $s$ & 2. & -1.24 \\
\hline 162.20 & $18-4,58$ & N. pach. $s$ & & -1.56 \\
\hline 165.12 & $18-5,42 \mathrm{~cm}$ & N. pach. $s$ & & -0.51 \\
\hline 165.76 & $18-5,102 \mathrm{~cm}$ & N. pach. s & & -1.06 \\
\hline 165.96 & $18-5,122 \mathrm{~cm}$ & N. pach. $s$ & & -1.45 \\
\hline 166.16 & $18-5,142 \mathrm{c}$ & N. pach. $s$ & & -1.44 \\
\hline 166.6 & $18-6,4$ & N. pe & 1. & -1.14 \\
\hline 180 & & & 6 & -0 \\
\hline & & $\Lambda$ & & 2 \\
\hline & $30-$ & $s$ & & 0.83 \\
\hline 23 & $32-$ & N. $p$ & 2 & 0.39 \\
\hline 240.82 & $32-2,1$ & N. pach. $s$ & 2 & 0.97 \\
\hline 242.12 & $32-3$, & N. pach. $s$ & 3.26 & 0.76 \\
\hline & $32-4,1$ & N. pach. $s$ & 2.72 & 0.81 \\
\hline & $33-1,122 \mathrm{~cm}$ & N. pach. $s$ & 2.60 & 0.57 \\
\hline 245.12 & $34-1,142 \mathrm{~cm}$ & N. pach. $s$ & 2.39 & 0.65 \\
\hline 248.72 & $34-2,122 \mathrm{~cm}$ & N. pach. $s$ & 2.48 & -0.21 \\
\hline 252.00 & $34-5,102 \mathrm{~cm}$ & N. pach. $s$ & 2.76 & 0.92 \\
\hline & $34-5,142$ & N. pach. $s$ & 2.04 & 0.92 \\
\hline & 34 & $N$ & 2.26 & 0.81 \\
\hline 253.40 & $34-6,142 \mathrm{~cm}$ & N. pach. $s$ & 2.99 & 1.04 \\
\hline
\end{tabular}

Shackleton et al. (1984) indicates relatively large glacials between 2.4 and $2.2 \mathrm{Ma}$. However, these are not as easily defined in the planktonic record. One reason for this discrepancy might be that colder deep-water temperatures in the North Atlantic during these glacials increased the amplitude of the benthic signal in addition to global ice volume changes. This seems to have occurred during the last glacial, probably due to deep-water formation within the North Atlantic region (Duplessy et al., 1980; Duplessy, 1982; Labeyrie et al., 1987) and might be a factor that has amplified isotopic variations also in earlier isotopic stages. The period between 2.0 and $1.2 \mathrm{Ma}$ was characterized by relatively small glacial episodes, as evidenced both by the Norwegian Sea and North Atlantic records. Throughout the period studied, the absolute benthic $\delta^{18} \mathrm{O}$ values of the North Atlantic were lighter than in the Norwegian Sea. Both records are adjusted to oxygen isotopic equilibrium, thus the difference signals permanently colder deep waters in the Norwegian Sea than in the North Atlantic.

\section{Carbonate Stratigraphy}

In Figure 6 carbonate records for Norwegian Sea Hole 642B and the carbonate stratigraphy for North Atlantic Hole 610A are plotted on equivalent time scales. The character of the carbonate signals differ markedly. The North Atlantic curve shows a pattern of alternating carbonate maxima and minima, primarily formed by carbonate dilution by varying ice-rafted debris (IRD) inputs, whereas the Norwegian Sea record reveals a pro-
Table 4. Benthic foraminiferal isotope data from Hole 644A. Here, ct $=$ Cassidulina teretis, $\mathbf{n b}=$ Melonis barleeanum.

\begin{tabular}{|c|c|c|c|c|}
\hline $\begin{array}{l}\text { Depth } \\
\text { (mbsf) }\end{array}$ & $\begin{array}{l}\text { Core/sect } \\
104-644 \mathrm{~A}-\end{array}$ & Species & $\delta^{18} \mathrm{O}$ & $\delta^{13} \mathrm{C}$ \\
\hline 1.92 & $1-2,42 \mathrm{~cm}$ & $\mathrm{ct}$ & 4.88 & -0.76 \\
\hline 2.72 & $1-2,122 \mathrm{~cm}$ & ct & 4.60 & -1.41 \\
\hline 4.22 & $1-3,122 \mathrm{~cm}$ & $\mathrm{ct}$ & 4.80 & -0.86 \\
\hline 6.42 & $1-5,42 \mathrm{~cm}$ & $\mathrm{nb}$ & 4.91 & -1.80 \\
\hline 6.82 & $1-5,83 \mathrm{~cm}$ & $\mathrm{nb}$ & 4.58 & -2.18 \\
\hline 9.62 & $2-1,42 \mathrm{~cm}$ & nb & 4.78 & -2.04 \\
\hline 10.00 & $2-1,82 \mathrm{~cm}$ & $\mathrm{nb}$ & 4.72 & -2.25 \\
\hline 15.62 & $2-5,42 \mathrm{~cm}$ & ct & 5.42 & -0.81 \\
\hline 17.22 & $3-1,102 \mathrm{~cm}$ & $\mathrm{ct}$ & 5.27 & -0.84 \\
\hline 18.96 & $3-2,122 \mathrm{~cm}$ & $\mathrm{nb}$ & 4.57 & -2.12 \\
\hline 26.32 & $4-1,62 \mathrm{~cm}$ & ct & 5.22 & -0.95 \\
\hline 26.72 & $4-1,102 \mathrm{~cm}$ & ct & 4.46 & -1.05 \\
\hline 27.12 & $4-1,142 \mathrm{~cm}$ & nb & 4.21 & 0.92 \\
\hline 28.02 & $4-2,82 \mathrm{~cm}$ & $\mathrm{ct}$ & 5.08 & -0.82 \\
\hline 31.02 & $4-4,82 \mathrm{~cm}$ & ct & 5.25 & -1.10 \\
\hline 31.92 & $4-5,22 \mathrm{~cm}$ & ct & 5.27 & -1.93 \\
\hline 31.92 & $4-5,22 \mathrm{~cm}$ & $\mathrm{nb}$ & 4.64 & -2.07 \\
\hline 39.92 & $5-4,22 \mathrm{~cm}$ & $\mathrm{ct}$ & 5.49 & -1.37 \\
\hline 46.42 & $6-2,22 \mathrm{~cm}$ & $\mathrm{nb}$ & 4.67 & -1.31 \\
\hline 47.40 & $6-2,122 \mathrm{~cm}$ & $\mathrm{nb}$ & 4.65 & -1.96 \\
\hline 50.21 & $6-4,101 \mathrm{~cm}$ & $\mathrm{ct}$ & 4.73 & -1.05 \\
\hline 51.29 & $6-5,59 \mathrm{~cm}$ & $\mathrm{ct}$ & 5.31 & -1.24 \\
\hline 53.21 & $6-6,101 \mathrm{~cm}$ & ct & 4.50 & -0.99 \\
\hline 63.91 & $8-1,23 \mathrm{~cm}$ & ct & 5.34 & -1.28 \\
\hline 65.60 & $8-2,40 \mathrm{~cm}$ & $\mathrm{ct}$ & 4.44 & -2.01 \\
\hline 66.90 & $8-3,20 \mathrm{~cm}$ & ct & 5.34 & -1.39 \\
\hline 67.37 & $8-3,60 \mathrm{~cm}$ & $\mathrm{ct}$ & 4.93 & -1.14 \\
\hline 68.10 & $8-3,142 \mathrm{~cm}$ & $\mathrm{nb}$ & 3.79 & -2.18 \\
\hline 71.12 & $8-5,142 \mathrm{~cm}$ & $\mathrm{ct}$ & 4.94 & -0.86 \\
\hline 71.42 & $8-6,22 \mathrm{~cm}$ & $\mathrm{nb}$ & 5.16 & -1.08 \\
\hline 71.80 & $8-6,60 \mathrm{~cm}$ & $\mathrm{nb}$ & 5.16 & -1.36 \\
\hline 73.79 & $9-1,59 \mathrm{~cm}$ & ct & 4.95 & -1.45 \\
\hline 74.02 & $9-1,82 \mathrm{~cm}$ & $\mathrm{nb}$ & 4.56 & -2.19 \\
\hline 74.22 & $9-1,102 \mathrm{~cm}$ & $\mathrm{ct}$ & 5.06 & -1.33 \\
\hline 74.43 & $9-1,123 \mathrm{~cm}$ & $\mathrm{ct}$ & 4.59 & -1.17 \\
\hline 74.43 & $9-1,123 \mathrm{~cm}$ & nb & 4.67 & -1.16 \\
\hline 75.93 & $9-2,123 \mathrm{~cm}$ & $\mathrm{ct}$ & 6.00 & -0.24 \\
\hline 76.43 & $9-3,23 \mathrm{~cm}$ & ct & 5.18 & -1.67 \\
\hline 76.60 & $9-3,40 \mathrm{~cm}$ & $\mathrm{ct}$ & 4.72 & -1.76 \\
\hline 77.02 & $9-3,82 \mathrm{~cm}$ & $\mathrm{nb}$ & 4.40 & -1.82 \\
\hline 77.22 & $9-3,102 \mathrm{~cm}$ & ct & 4.90 & -1.43 \\
\hline 77.43 & $9-3,123 \mathrm{~cm}$ & $\mathrm{ct}$ & 4.75 & -1.31 \\
\hline 79.02 & $9-4,82 \mathrm{~cm}$ & $\mathrm{ct}$ & 5.14 & -1.28 \\
\hline 84.61 & $10-2,41 \mathrm{~cm}$ & $\mathrm{nb}$ & 3.79 & -2.28 \\
\hline 84.78 & $10-2,58 \mathrm{~cm}$ & $\mathrm{ct}$ & 4.59 & -1.19 \\
\hline 85.42 & $10-2,122 \mathrm{~cm}$ & ct & 6.04 & -0.55 \\
\hline 85.92 & $10-3,21 \mathrm{~cm}$ & ct & 4.82 & -0.79 \\
\hline 86.11 & $10-3,41 \mathrm{~cm}$ & $\mathrm{nb}$ & 4.29 & -2.58 \\
\hline 86.48 & $10-3,78 \mathrm{~cm}$ & $\mathrm{ct}$ & 4.41 & -1.32 \\
\hline 88.91 & $10-5,22 \mathrm{~cm}$ & ct & 5.36 & -1.60 \\
\hline 89.28 & $10-5,58 \mathrm{~cm}$ & ct & 5.12 & -0.82 \\
\hline 89.48 & $10-5,78 \mathrm{~cm}$ & ct & 5.27 & -0.84 \\
\hline 93.62 & $11-1,142 \mathrm{~cm}$ & $\mathrm{nb}$ & 4.42 & -2.12 \\
\hline 94.09 & $11-2,39 \mathrm{~cm}$ & $\mathrm{ct}$ & 4.46 & -1.30 \\
\hline 94.33 & $11-2,63 \mathrm{~cm}$ & ct & 4.34 & -1.40 \\
\hline 94.92 & $11-2,122 \mathrm{~cm}$ & $\mathrm{ct}$ & 4.43 & -0.89 \\
\hline 95.42 & $11-3,22 \mathrm{~cm}$ & ct & 4.62 & -1.99 \\
\hline 97.51 & $11-4,80 \mathrm{~cm}$ & ct & 4.97 & -1.84 \\
\hline 98.42 & $11-5,22 \mathrm{~cm}$ & ct & 4.81 & -1.78 \\
\hline 99.92 & $11-6,22 \mathrm{~cm}$ & $\mathrm{ct}$ & 4.07 & -1.88 \\
\hline 100.30 & $11-6,60 \mathrm{~cm}$ & $\mathrm{ct}$ & 4.63 & -1.40 \\
\hline 100.58 & $11-6,88 \mathrm{~cm}$ & ct & 4.38 & -2.08 \\
\hline 100.70 & $11-6,100 \mathrm{~cm}$ & $c t$ & 3.77 & -2.21 \\
\hline 100.92 & $11-6,122 \mathrm{~cm}$ & ct & 4.41 & -0.79 \\
\hline \multirow[t]{2}{*}{101.14} & $11-6,142 \mathrm{~cm}$ & ct & 4.25 & -1.59 \\
\hline & $11-7,22 \mathrm{~cm}$ & ct & 4.54 & -1.82 \\
\hline 102.12 & $12-1,39 \mathrm{~cm}$ & $\mathrm{nb}$ & 4.27 & -2.01 \\
\hline 102.32 & $12-1,64 \mathrm{~cm}$ & $\mathrm{nb}$ & 4.18 & -2.00 \\
\hline 102.52 & $12-1,82 \mathrm{~cm}$ & ct & 4.59 & -0.89 \\
\hline 102.72 & $12-1,102 \mathrm{~cm}$ & ct & 4.87 & -0.94 \\
\hline 102.72 & $12-1,10 \mathrm{~cm}$ & $\mathrm{nb}$ & 4.38 & -1.69 \\
\hline 104.40 & $12-2,122 \mathrm{~cm}$ & nb & 4.36 & -1.91 \\
\hline 106.42 & $12-4,22 \mathrm{~cm}$ & $\mathrm{ct}$ & 4.65 & -1.08 \\
\hline 106.62 & $12-4,42 \mathrm{~cm}$ & ct & 4.73 & -1.14 \\
\hline 106.63 & $12-4,62 \mathrm{~cm}$ & $\mathrm{ct}$ & 4.82 & -1.30 \\
\hline
\end{tabular}


Table 4 (continued).

\begin{tabular}{|c|c|c|c|c|}
\hline $\begin{array}{l}\text { Depth } \\
\text { (mbsf) }\end{array}$ & $\begin{array}{l}\text { Core/sect } \\
104-644 \mathrm{~A}-\end{array}$ & Species & $\delta^{18} \mathrm{O}$ & $\delta^{13} \mathrm{C}$ \\
\hline 110.02 & $12-6,82 \mathrm{~cm}$ & $\mathrm{ct}$ & 4.47 & -1.81 \\
\hline 110.22 & $12-6,102 \mathrm{~cm}$ & nb & 3.89 & -2.59 \\
\hline 110.60 & $12-6,142 \mathrm{~cm}$ & $\mathrm{nb}$ & 3.69 & -2.28 \\
\hline 110.92 & $12-7,22 \mathrm{~cm}$ & $\mathrm{ct}$ & 4.12 & -1.66 \\
\hline 111.82 & $13-1,62 \mathrm{~cm}$ & nb & 3.95 & -2.17 \\
\hline 111.82 & $13-00,21 \mathrm{~cm}$ & $\mathrm{ct}$ & 4.90 & -1.09 \\
\hline 112.02 & $13-1,82 \mathrm{~cm}$ & $\mathrm{ct}$ & 4.29 & -2.05 \\
\hline 112.42 & $13-1,122 \mathrm{~cm}$ & $\mathrm{ct}$ & 4.39 & -1.57 \\
\hline 112.60 & $13-1,142 \mathrm{~cm}$ & $\mathrm{ct}$ & 4.75 & -1.60 \\
\hline 113.32 & $13-2,62 \mathrm{~cm}$ & $\mathrm{ct}$ & 4.90 & -1.33 \\
\hline 113.52 & $13-2,82 \mathrm{~cm}$ & $\mathrm{ct}$ & 4.96 & -1.12 \\
\hline 114.41 & $13-3,21 \mathrm{~cm}$ & $\mathrm{ct}$ & 5.25 & -0.95 \\
\hline 115.91 & $13-4,21 \mathrm{~cm}$ & $\mathrm{ct}$ & 4.89 & -1.58 \\
\hline 116.12 & $13-4,42 \mathrm{~cm}$ & $\mathrm{ct}$ & 5.34 & -1.38 \\
\hline 116.32 & $13-4,62 \mathrm{~cm}$ & $\mathrm{ct}$ & 5.20 & -1.22 \\
\hline 116.72 & $13-4,102 \mathrm{~cm}$ & $\mathrm{ct}$ & 4.86 & -1.62 \\
\hline 117.41 & $13-5,21 \mathrm{~cm}$ & $\mathrm{ct}$ & 4.74 & -1.67 \\
\hline 117.62 & $13-5,42 \mathrm{~cm}$ & nb & 4.60 & -1.61 \\
\hline 118.91 & $13-6,21 \mathrm{~cm}$ & ct & 5.10 & -1.49 \\
\hline 119.12 & $13-6,42 \mathrm{~cm}$ & $\mathrm{ct}$ & 4.88 & -1.60 \\
\hline 119.32 & $13-6,62 \mathrm{~cm}$ & $\mathrm{ct}$ & 4.70 & -1.86 \\
\hline 119.52 & $13-6,82 \mathrm{~cm}$ & nb & 4.61 & -1.92 \\
\hline 120.41 & $13-7,21 \mathrm{~cm}$ & ct & 5.03 & -1.33 \\
\hline 120.82 & $13-7,65 \mathrm{~cm}$ & ct & 4.57 & -1.73 \\
\hline 120.91 & $14-1,20 \mathrm{~cm}$ & $\mathrm{ct}$ & 5.22 & -1.18 \\
\hline 121.22 & $13-7,102 \mathrm{~cm}$ & $\mathrm{ct}$ & 4.89 & -1.77 \\
\hline 121.42 & $13-7,122 \mathrm{~cm}$ & $\mathrm{ct}$ & 4.86 & -1.76 \\
\hline 123.32 & $14-3,62 \mathrm{~cm}$ & $\mathrm{ct}$ & 5.57 & -0.39 \\
\hline 126.02 & $14-4,82 \mathrm{~cm}$ & $\mathrm{ct}$ & 4.37 & -2.09 \\
\hline 126.91 & $14-5,21 \mathrm{~cm}$ & $\mathrm{ct}$ & 5.14 & -1.65 \\
\hline 127.72 & $14-5,103 \mathrm{~cm}$ & ct & 5.10 & -1.60 \\
\hline 128.11 & $14-5,142 \mathrm{~cm}$ & $\mathrm{ct}$ & 5.59 & -1.30 \\
\hline 130.42 & $15-1,22 \mathrm{~cm}$ & ct & 4.97 & -1.82 \\
\hline 130.62 & $15-1,42 \mathrm{~cm}$ & $\mathrm{ct}$ & 4.87 & -1.83 \\
\hline 130.82 & $15-1,62 \mathrm{~cm}$ & $\mathrm{ct}$ & 4.51 & -1.55 \\
\hline 131.02 & $15-1,82 \mathrm{~cm}$ & $\mathrm{nb}$ & 4.60 & -1.79 \\
\hline 131.42 & $15-1,12 \mathrm{~cm}$ & $\mathrm{ct}$ & 5.24 & -1.49 \\
\hline 131.42 & $15-1,122 \mathrm{~cm}$ & nb & 4.58 & -1.86 \\
\hline 133.62 & $15-3,62 \mathrm{~cm}$ & ct & 4.40 & -1.60 \\
\hline 134.02 & $15-3,102 \mathrm{~cm}$ & ct & 4.57 & -1.85 \\
\hline 134.22 & $15-3,122 \mathrm{~cm}$ & $\mathrm{ct}$ & 4.48 & -1.68 \\
\hline 134.42 & $15-3,142 \mathrm{~cm}$ & $\mathrm{ct}$ & 5.16 & -1.13 \\
\hline 136.42 & $15-5,22 \mathrm{~cm}$ & $\mathrm{ct}$ & 4.69 & -1.36 \\
\hline 136.82 & $15-4,62 \mathrm{~cm}$ & $\mathrm{ct}$ & 4.79 & -1.58 \\
\hline 137.02 & $15-5,82 \mathrm{~cm}$ & $\mathrm{ct}$ & 4.65 & -1.84 \\
\hline 137.22 & $15-5,102 \mathrm{~cm}$ & $\mathrm{ct}$ & 5.09 & -1.33 \\
\hline 137.42 & $15-5,122 \mathrm{~cm}$ & $\mathrm{ct}$ & 4.30 & -1.88 \\
\hline 137.62 & $15-5,142 \mathrm{~cm}$ & $\mathrm{ct}$ & 4.59 & -1.51 \\
\hline 142.02 & $16-2,102 \mathrm{~cm}$ & $\mathrm{ct}$ & 4.14 & -1.82 \\
\hline 142.20 & $16-2,120 \mathrm{~cm}$ & $\mathrm{ct}$ & 4.74 & -2.08 \\
\hline 142.42 & $16-2,142 \mathrm{~cm}$ & ct & 4.44 & -1.62 \\
\hline 143.12 & $16-3,42 \mathrm{~cm}$ & ct & 4.27 & -2.13 \\
\hline 145.02 & $16-4,102 \mathrm{~cm}$ & $\mathrm{ct}$ & 4.76 & -1.18 \\
\hline 145.20 & $16-4,120 \mathrm{~cm}$ & $\mathrm{ct}$ & 4.41 & -1.46 \\
\hline 145.93 & $16-5,23 \mathrm{~cm}$ & $\mathrm{ct}$ & 4.65 & -0.97 \\
\hline 146.72 & $16-5,102 \mathrm{~cm}$ & $\mathrm{ct}$ & 4.50 & -1.45 \\
\hline 146.92 & $16-5,120 \mathrm{~cm}$ & $\mathrm{ct}$ & 4.62 & -1.82 \\
\hline 147.12 & $16-5,142 \mathrm{~cm}$ & $\mathrm{ct}$ & 4.70 & -1.54 \\
\hline 150.92 & $17-2,22 \mathrm{~cm}$ & $\mathrm{ct}$ & 4.38 & -1.35 \\
\hline 151.12 & $17-2,42 \mathrm{~cm}$ & $\mathrm{ct}$ & 4.48 & -1.51 \\
\hline 151.32 & $17-2,62 \mathrm{~cm}$ & $\mathrm{ct}$ & 4.02 & -1.66 \\
\hline 152.42 & $17-3,22 \mathrm{~cm}$ & ct & 4.82 & -1.52 \\
\hline 152.62 & $17-3,42 \mathrm{~cm}$ & $\mathrm{ct}$ & 4.83 & -1.47 \\
\hline 152.82 & $17-3,62 \mathrm{~cm}$ & $\mathrm{ct}$ & 4.81 & -1.38 \\
\hline 154.12 & $17-4,42 \mathrm{~cm}$ & nb & 4.41 & -1.87 \\
\hline 154.32 & $17-4,102 \mathrm{~cm}$ & $\mathrm{ct}$ & 4.50 & -1.47 \\
\hline 154.52 & $17-4,122 \mathrm{~cm}$ & ct & 4.54 & -1.33 \\
\hline 154.72 & $17-4,142 \mathrm{~cm}$ & $\mathrm{ct}$ & 4.64 & -1.18 \\
\hline 155.42 & $17-5,22 \mathrm{~cm}$ & ct & 4.65 & $-1,30$ \\
\hline 156.42 & $17-5,122 \mathrm{~cm}$ & ct & 4.78 & -1.29 \\
\hline 159.52 & $18-1,102 \mathrm{~cm}$ & nb & 4.17 & -1.96 \\
\hline 159.72 & $18-1,122 \mathrm{~cm}$ & $\mathrm{ct}$ & 4.71 & -1.64 \\
\hline 159.92 & $18-1,142 \mathrm{~cm}$ & $\mathrm{ct}$ & 5.01 & -1.63 \\
\hline 162.12 & $18-3,42 \mathrm{~cm}$ & $\mathrm{ct}$ & 4.25 & -1.68 \\
\hline 162.52 & $18-3,102 \mathrm{~cm}$ & ct & 4.56 & -2.54 \\
\hline 162.72 & $18-3,122 \mathrm{~cm}$ & ct & 3.79 & -1.50 \\
\hline
\end{tabular}

Table 4 (continued).

\begin{tabular}{|c|c|c|c|c|}
\hline $\begin{array}{l}\text { Depth } \\
\text { (mbsf) }\end{array}$ & $\begin{array}{l}\text { Core/sect } \\
104-644 \mathrm{~A}-\end{array}$ & Species & $\delta^{18} \mathrm{O}$ & $\delta^{13} \mathrm{C}$ \\
\hline 163.62 & $18-4,42 \mathrm{~cm}$ & $\mathrm{ct}$ & 4.30 & -0.93 \\
\hline 163.80 & $18-4,58 \mathrm{~cm}$ & ct & 4.18 & -0.99 \\
\hline 165.12 & $18-5,42 \mathrm{~cm}$ & ct & 3.37 & -1.34 \\
\hline 165.30 & $18-5,58 \mathrm{~cm}$ & ct & 4.01 & -1.18 \\
\hline 165.76 & $18-5,102 \mathrm{~cm}$ & ct & 3.96 & -1.19 \\
\hline 165.96 & $18-5,12 \mathrm{~cm}$ & ct & 3.41 & -1.50 \\
\hline 165.96 & $18-5,122 \mathrm{~cm}$ & nb & 4.26 & -1.99 \\
\hline 166.16 & $18-5,142 \mathrm{~cm}$ & $\mathrm{ct}$ & 3.48 & -1.83 \\
\hline 166.62 & $18-6,42 \mathrm{~cm}$ & ct & 4.41 & -1.69 \\
\hline 166.80 & $18-6,58 \mathrm{~cm}$ & $\mathrm{nb}$ & 4.58 & -2.04 \\
\hline 168.30 & $18-7,58 \mathrm{~cm}$ & $\mathrm{ct}$ & 4.11 & -1.13 \\
\hline 169.92 & $19-2,21 \mathrm{~cm}$ & $\mathrm{nb}$ & 4.35 & -2.24 \\
\hline 171.42 & $19-3,22 \mathrm{~cm}$ & ct & 4.71 & -1.59 \\
\hline 172.02 & $19-3,82 \mathrm{~cm}$ & ct & 4.63 & -1.65 \\
\hline 172.18 & $19-3,98 \mathrm{~cm}$ & ct & 4.49 & -1.72 \\
\hline 172.40 & $19-3,121 \mathrm{~cm}$ & $\mathrm{ct}$ & 4.06 & -1.92 \\
\hline 172.62 & $19-3,142 \mathrm{~cm}$ & ct & 4.59 & -1.49 \\
\hline 172.92 & $19-4,22 \mathrm{~cm}$ & ct & 4.70 & -1.47 \\
\hline 173.52 & $19-4,82 \mathrm{~cm}$ & ct & 4.84 & -1.07 \\
\hline 173.70 & $19-4,100 \mathrm{~cm}$ & $\mathrm{ct}$ & 4.68 & -1.18 \\
\hline 178.12 & $20-1,142 \mathrm{~cm}$ & nb & 4.66 & -2.13 \\
\hline 178.42 & $20-1,102 \mathrm{~cm}$ & $\mathrm{ct}$ & 4.66 & -1.29 \\
\hline 178.62 & $20-1,122 \mathrm{~cm}$ & ct & 4.66 & -1.73 \\
\hline 178.62 & $20-1,142 \mathrm{~cm}$ & ct & 4.83 & -1.47 \\
\hline 179.92 & $19-4,21 \mathrm{~cm}$ & $\mathrm{nb}$ & 4.28 & -1.96 \\
\hline 180.00 & $20-2,102 \mathrm{~cm}$ & ct & 4.68 & -1.63 \\
\hline 180.20 & $20-2,122 \mathrm{~cm}$ & $\mathrm{ct}$ & 4.46 & -1.93 \\
\hline 180.40 & $20-2,142 \mathrm{~cm}$ & $\mathrm{ct}$ & 4.64 & -1.68 \\
\hline 181.30 & $20-3,82 \mathrm{~cm}$ & ct & 5.40 & -0.73 \\
\hline 181.50 & $20-3,102 \mathrm{~cm}$ & ct & 5.01 & -1.16 \\
\hline 181.70 & $20-3,122 \mathrm{~cm}$ & ct & 4.88 & -1.11 \\
\hline 182.60 & $20-4,42 \mathrm{~cm}$ & $\mathrm{ct}$ & 4.93 & -1.29 \\
\hline 184.10 & $20-5,42 \mathrm{~cm}$ & ct & 4.47 & -1.02 \\
\hline 184.62 & $21-1,22 \mathrm{~cm}$ & ct & 4.47 & -0.59 \\
\hline 187.00 & $22-1,22 \mathrm{~cm}$ & ct & 4.93 & -0.85 \\
\hline 187.20 & $22-1,42 \mathrm{~cm}$ & ct & 5.18 & -0.93 \\
\hline 187.80 & $22-1,102 \mathrm{~cm}$ & $\mathrm{ct}$ & 4.58 & -1.35 \\
\hline 188.90 & $22-2,62 \mathrm{~cm}$ & ct & 5.05 & -1.00 \\
\hline 191.90 & $22-4,62 \mathrm{~cm}$ & ct & 4.76 & -1.25 \\
\hline 192.10 & $22-4,102 \mathrm{~cm}$ & ct & 4.61 & -1.04 \\
\hline 196.00 & $23-2,42 \mathrm{~cm}$ & $\mathrm{ct}$ & 4.33 & -1.00 \\
\hline 196.60 & $23-2,102 \mathrm{~cm}$ & $\mathrm{ct}$ & 4.54 & -0.94 \\
\hline 196.80 & $23-2,122 \mathrm{~cm}$ & $\mathrm{ct}$ & 4.52 & -0.96 \\
\hline 197.50 & $23-3,42 \mathrm{~cm}$ & ct & 4.57 & -0.94 \\
\hline 201.30 & $23-5,120 \mathrm{~cm}$ & ct & 4.75 & -0.58 \\
\hline 201.52 & $23-5,142 \mathrm{~cm}$ & ct & 4.63 & -0.43 \\
\hline 205.12 & $24-3,102 \mathrm{~cm}$ & ct & 4.43 & -1.08 \\
\hline 205.20 & $24-4,20 \mathrm{~cm}$ & ct & 4.21 & -1.09 \\
\hline 206.20 & $25-1,22 \mathrm{~cm}$ & ct & 4.49 & -1.18 \\
\hline 207.70 & $25-2,22 \mathrm{~cm}$ & $\mathrm{ct}$ & 4.31 & -1.16 \\
\hline 208.12 & $25-2,62 \mathrm{~cm}$ & $\mathrm{ct}$ & 4.50 & -0.72 \\
\hline 212.60 & $26-2,62 \mathrm{~cm}$ & ct & 4.89 & -0.79 \\
\hline 239.12 & $32-1,102 \mathrm{~cm}$ & ct & 5.13 & -0.01 \\
\hline 240.82 & $32-2,122 \mathrm{~cm}$ & $\mathrm{ct}$ & 4.34 & -0.67 \\
\hline 242.12 & $32-3,102 \mathrm{~cm}$ & ct & 4.61 & -0.95 \\
\hline 243.60 & $32-4,102 \mathrm{~cm}$ & ct & 4.55 & -0.87 \\
\hline 244.82 & $33-1,122 \mathrm{~cm}$ & $\mathrm{ct}$ & 3.89 & -1.39 \\
\hline 248.72 & $34-2,122 \mathrm{~cm}$ & ct & 3.91 & -1.55 \\
\hline 252.00 & $34-5,102 \mathrm{~cm}$ & ct & 3.94 & -1.49 \\
\hline 252.22 & $34-5,122 \mathrm{~cm}$ & ct & 3.87 & -1.21 \\
\hline 252.40 & $34-5,142 \mathrm{~cm}$ & ct & 4.72 & -0.48 \\
\hline 253.20 & $34-6,122 \mathrm{~cm}$ & $\mathrm{ct}$ & 4.22 & -1.26 \\
\hline 253.40 & $34-6,142 \mathrm{~cm}$ & $\mathrm{ct}$ & 4.19 & -1.06 \\
\hline
\end{tabular}

longed period of essentially no carbonate through most of the Matuyama. The study of glacial lithologies and calcite dissolution by Henrich (this volume) proposes that the reason for this difference is widespread calcite dissolution during extended time intervals in the Norwegian Sea. Henrich (this volume) presented a model in which carbonate dissolution is thought to be enhanced during periods of reduced deep-water ventilation, probably due to reduced surface salinities that stabilize the water col- 


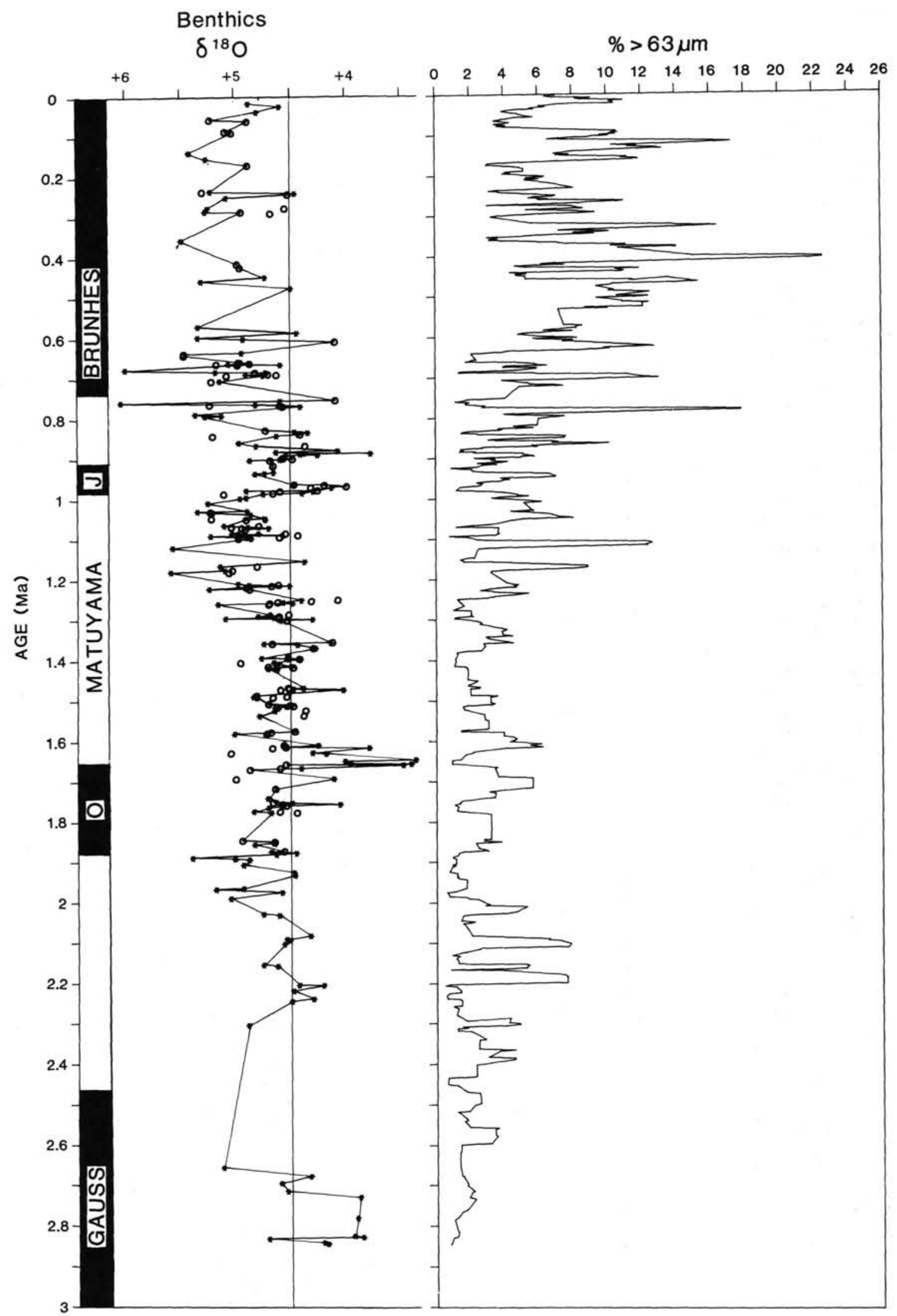

Figure 5. Coarse-fraction and isotope record of Hole 644A. The coarse-fraction record was filtered by applying a 5-point moving average filter to avoid high-frequency variation, to better evaluate long-term patterns. 


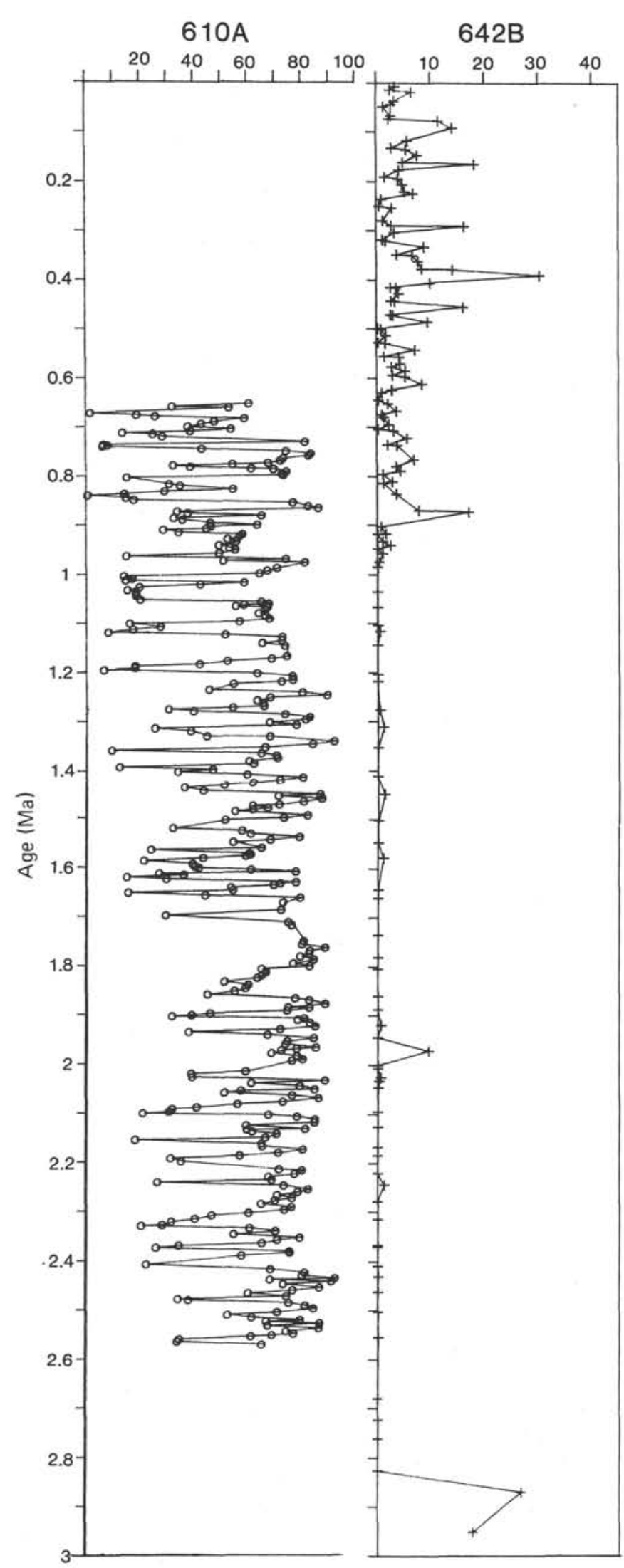

Figure 6. Carbonate records from Hole $610 \mathrm{~A}$ in the North Atlantic and $642 \mathrm{~B}$ in the Norwegian Sea, plotted on the same time scale. Paleomagnetic reversals were used as fixing points for the time scale. Each data point was placed based on linear sedimentation rates between fixed points. umn and prevent vertical overturn. This in turn leads to an increase in $\mathrm{P}_{\mathrm{CO}_{2}}$ of bottom water associated with low carbonate productivity. Analogs for this model are found in similar glacial-interglacial lithofacies of the upper Quaternary, however, where dissolution coincides with deglaciation. In contrast, the entire Matuyama of Hole 642B and large parts of Hole 644A are marked by a strong dissolution event (Henrich, 1986, Henrich, this volume). Between the upper Quaternary pattern and the Matuyama dissolution pattern is a relatively long transition period from about 0.9 to $0.6 \mathrm{Ma}$, marked by swift changes between preservation and dissolution (Henrich, this volume). This interval coincides with the transition period between light and heavy planktonic $\delta^{18} \mathrm{O}$ values and reduced surface- to deep-water $\delta^{18} \mathrm{O}$ gradient (Figs. 3 and 4 ).

\section{SEDIMENTOLOGICAL EVIDENCE FOR GLACIAL EVENTS}

In Figure 7 the weight percentage of the coarse fraction in Hole $644 \mathrm{~A}$ is plotted vs. age. To emphasize the major features of the coarse-fraction record, we smoothed the records with a 5 -point running average filter that eliminates some high-frequency perturbations. The filtered record is shown beside the oxygen isotope record from this hole, plotted against the magnetic time scale. High-frequency peaks are evident throughout the hole, starting at 233 meters below seafloor (mbsf) $(2.56$ Ma), slightly beneath the Matuyama/Gauss boundary. The first evidence of glacial lithologies is also found at this stratigrahic position at Site 642, which extends much further back in time, thereby confirming the first appearance in Hole 644A (Henrich, this volume). Visual examination of the samples shows that the dominant coarse-fraction component of the section above 233 mbsf is terrigenous sand, composed of quartz, feldspar, and rock fragments. This evidence and the close correspondence between coarse-fraction peaks and glaciomarine lithologies (Henrich this volume), suggest that the coarse-fraction variation basically reflects ice-rafted input to the area.

Based on the paleomagnetic time scale, the first major input of IRD appears at about $2.6 \mathrm{Ma}(2.56 \mathrm{Ma}$ is the best estimate from simple extrapolation), and since then glacial-type environments have characterized the region. Larger inputs of IRD occur at 2.0-2.2 Ma, and 2.3-2.4 Ma. Inputs were low from 2.0 $\mathrm{Ma}$ and until about 1.2 Ma. However, the sediments from this period are glacial in nature. Between 1.2 and $0.6 \mathrm{Ma}$, IRD inputs were moderate. From $0.6 \mathrm{Ma}$ to the present, input of IRD increased such that the maxima are of greater magnitude and duration. The signal of the fraction $>63 \mu \mathrm{m}$ is similar to that observed in isotopic and carbonate records. That is, the same pattern of relative quiescence occurs during much of the Matuyama, an intermediate phase during the late Matuyama and the early Brunhes, and with stronger signals in the rest of the Brunhes. We believe this quiescence to reflect general trends in the development of the climate and ocean system of the Norwegian Sea. This pattern may extend to the global climate system as well.

The close proximity of Site 644 to the Norwegian mainland implies that the IRD fluctuations reflect the waxing and waning of the Scandinavian ice sheet. Large IRD input reflects largescale deglaciations of marine based portions of the ice sheet. With a more eastern deflection of the East Greenland current, it is also possible for icebergs to enter the Vøring Plateau from the west. This would affect Site 643 on the outer Vøring Plateau and constitute a less-likely source for the IRD found at Site 644 on the inner Vøring Plateau.

\section{PREGLACIAL ENVIRONMENTS AND ONSET OF LARGE-SCALE GLACIATION IN SCANDINAVIA}

At Sites 642 and 644, the first evidence of glacial lithologies is found immediately beneath the Matuyama/Gauss boundary 

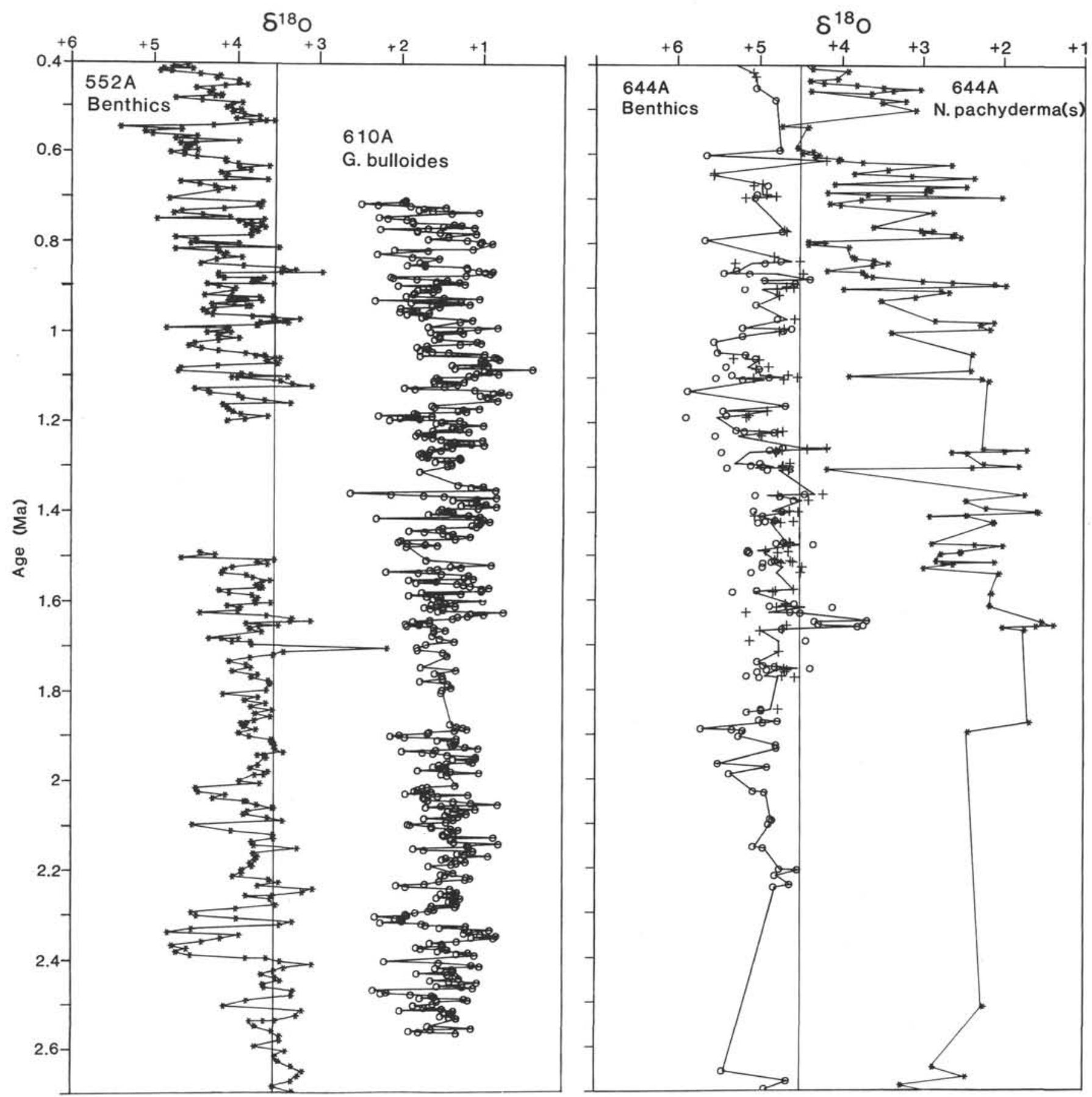

Figure 7. Planktonic and benthic oxygen isotope records from the North Atlantic and the Norwegian Sea. The time scale was produced as described for Figure 6. The record from Hole 552A was taken from Shackleton and Hall (1984). The vertical lines in the benthic oxygen isotope records refer to Holocene equilibrium values.

at about $2.56 \mathrm{Ma}$, based on linear extrapolation from the Matuyama/Gauss boundary. This is somewhat older than the age of $2.36 \mathrm{Ma}$ reported for the first major ${ }^{18} \mathrm{O}$ enrichment from Site 552 in the North Atlantic (Shackleton et al., 1984). However, Shackleton et al. (op cit.) also note a short pulse of ice-rafting shortly before the Gauss/Matuyama boundary at about $2.6 \mathrm{Ma}$. In a study of Site 606 in the North Atlantic, Keigwin (1967) concluded that the late Pliocene was characterized by a series of ${ }^{18} \mathrm{O}$ enrichments dated at about 3.1, 2.7, 2.6, and $2.4 \mathrm{Ma}$ based on magnetostratigraphy. The two younger events correlate to lithologic and isotopic evidence for glaciation both from Site 552
(Shackleton et al., 1984) and from this study. All the Leg 104 sites document major Neogene glacial expansions evidenced by increased inputs of coarse-grained terrigenous material and a marked lithological change from biogenous sediments to repeated successions of glacial marine lithologies at $2.56 \mathrm{Ma}$ (Henrich, this volume, Jansen et al., this volume). A discrepancy exists, therefore, these records and reports of earlier glacial phases at $3 \mathrm{Ma}$ on Iceland (Einarsson et al., 1967) and in the Arctic Ocean in the late Miocene (Clark, 1982). However, data under preparation from a detailed study of the late Miocene-Pliocene interval in Hole 642B may indicate glaciation somewhere around the 
Norwegian-Greenland Sea as early as $5.4 \mathrm{Ma}$ (E. Jansen, preliminary unpublished data). However, as shown by the sedimentological studies, the major shift to large and repeated glacial cycles took place at about $2.6 \mathrm{Ma}$.

The carbon isotope values of the preglacial period in the Norwegian Sea are relatively positive, indicating open surface waters and ventilated deep waters. Open surface waters is also indicated by the diverse planktonic foraminifer assemblages prior to the glacial onset (Spiegler and Jansen, this volume). Although the $\delta{ }^{18} \mathrm{O}$ data are few in Hole 644A they indicate that also in the preglacial period the deep waters of the Norwegian sea were colder than their North Atlantic counterparts (i.e., heavier $\delta^{18} \mathrm{O}$ ). Thus deep water formation in the Norwegian-Greenland Sea contributed to the production NADW both before and after the major glacial onset at $2.56 \mathrm{Ma}$.

\section{PALEOENVIRONMENTS 2.6-0.73 Ma}

The coarse-fraction data indicate relatively large glaciations at 2.2 and $2.1 \mathrm{Ma}$. The period between approximately 2.0 and 1.2 Ma was marked by low coarse-fraction input, indicating less extensive glaciation. The oxygen isotope record of this time is characterized by low-amplitude fluctuations (Figs. 3 and 5). The benthic oxygen isotope values from Hole 644A during Matuyama interglacials are similar to Holocene values, suggesting (Fig. 3) the same global ice volume as today during interglacials of the Matuyama. The sedimentologic records also show rapidly alternating environments with shifting lithologies. However, the coarse-fraction throughout the period 2.5 to $0.9 \mathrm{Ma}$ is almost entirely composed of terrigenous sand (IRD) and also in intervals where oxygen isotopes indicate low ice volume. Furthermore, the lack of carbonate, the widespread dissolution (Henrich, this volume) and the fact that only polar planktonic foraminifers are found from this period (Spiegler and Jansen, this volume) suggest that most of the Matuyama was characterized by significantly colder climates in the Norwegian Sea than today. The cold or glacial stadials were much more restricted than those of the later periods locally in northern Europe and globally. The data suggest that the climatic variation of the Norwegian Sea during the period 2.5 to $1.2 \mathrm{Ma}$ was characterized by generally cool conditions where climates shifted within a rather narrow range of cold acrtic environments with much lower amplitudes than the late Pleistocene glacial-to-interglacial cycles.

These data indicate that the thermal gradient between the Norwegian Sea and the North Atlantic was much stronger during the Matuyama than at present. The North Atlantic surface current system was probably deflected more to the east and south during the Matuyama and the influx of temperate Atlantic water through the Norwegian Current was rather weak. A more zonal climatic system than today would probably account for an increased thermal gradient between the Norwegian Sea and the North Atlantic, and could also promote cooler, less saline surface waters in the Norwegian Sea. A tentative model would include a more westerly wind system with reduced frequency of northward moving storms through the Icelandic Low, in contrast to the present meridional type of climate over the Norwegian Sea. More continuous ice- and snow-cover over both land and sea would be expected from a stronger thermal gradient between the North Atlantic and the Norwegian Sea and would in return further promote zonality. Ruddiman et al. (1986b) showed that North Atlantic SST variation during this period is solely dominated by Earth's 41,000 -yr obliquity period and that the thermal response is in phase with $\delta^{18} \mathrm{O}$ signals and hence with ice-sheet variations. This probably indicates that SST variations in the Matuyama mainly reflect the size of the Northern Hemisphere ice sheets. In a discussion of the climatic shifts near 2.5 and $0.9 \mathrm{Ma}$, Ruddiman et al. (1986b) propose that the shift from a 41,000 -yr cyclicity to a dominant 100,000 -yr cyclic- ity at $0.9 \mathrm{Ma}$ might have been caused by increased meridionality of the Northern Hemisphere climate system. This seems to be supported by our data. Climate models have shown that tectonic uplift and mountain building in the Himalayas/Tibet and western North America may lead to increased meridionality (Manabe and Terpstra, 1974, Grose and Hoskins, 1979).

The strong calcite dissolution of the Matuyama period in the Norwegian Sea is an indication of high deep water $\mathrm{P}_{\mathrm{CO}_{2}}$ probably caused by reduced deep-water ventilation (Henrich, this volume). Reduced ventilation and high nutrient levels of subsurface waters are indicated by the more negative carbon isotope values in N. pachyderma during this period (Table 1, Figs. 3 and 4). There are no indications of anoxia, however. The heavier benthic oxygen isotopes in the Norwegian Sea indicate that deep waters were colder than in the North Atlantic during the Matuyama. Some type of deep water formation within the NorwegianGreenland Sea/Arctic Ocean is required to explain this difference. This might not necessarily mean deep-water formation brought about by open ocean surface cooling. Other mechanisms such as formation of dense winter waters on shelves or as a response to sea-ice formation (Aagard et al., 1985) can also be considered. To what extent the Norwegian Sea contributed to NADW is difficult to assess. The North Atlantic benthic $\delta^{13} \mathrm{C}$ record clearly shows that NADW throughout this period was better ventilated than the Pacific (Shackleton et al., 1984, Shackleton and Hall, 1984), which suggests a source of ventilated deep water somewhere in the North Atlantic/Norwegian Sea region. A direct comparison of benthic $\delta^{13} \mathrm{C}$ between the Norwegian Sea and the North Atlantic is not possible because the factors influencing carbon isotopic disequilibria for the species used in the present study are not properly understood. Studies of the last glacial indicate that the North Atlantic can produce its own deep water under certain conditions (Duplessy et al., 1980; Labeyrie and Duplessy, 1985; Mix and Fairbanks, 1985; Labeyrie et al., 1987; Zahn, 1986), but available data cannot resolve whether glacial deep water formation was active during earlier time intervals.

During the last few years much attention has been focused on the climatic shift at about $0.9 \mathrm{Ma}$. This appears in different paleoclimatic time series as a change toward larger climatic amplitudes and lower frequency oscillation (Shackleton and Opdyke 1973, Prell 1982). In sea-surface records from the North Atlantic the shift marks a change toward 100-k.y. dominance in climatic spectra (Ruddiman et al., 1986a, b). Different parameters from the Norwegian Sea also evidence a change centered around $0.9 \mathrm{Ma}$. The oxygen isotopic gradient between deep and surface waters decreased, inputs of IRD increased, and carbonate dissolution ceased (Henrich, this volume). These changes indicate a change toward more extensive glacials including longer durations and the formation of a larger Scandinavian ice sheet. They also mark the initiation of warmer interglacials with warm surface-water influx and ventilated deep waters similar to the Holocene. Although the change appears to be centered at 0.9 $\mathrm{Ma}$, the change in the different parameters is of a gradual rather than sudden type. The shift begins at about $1.2 \mathrm{Ma}$ and ends at about $0.6 \mathrm{Ma}$. This supports the suggestion that global climatic change was gradual (Ruddiman et al., 1986a) caused by changes in climatic boundary conditions that required time to develop.

Although the available European continental and shelf records are more episodic and more poorly dated than the records from Leg 104, they agree reasonably well with our data. The record from the Netherlands show evidence for considerable cooling at the Gauss/Matuyama boundary, in agreement with the first glacial evidence in the Norwegian Sea sediments. This cold phase was followed by a complex series of temperate and cool stages based on palynological indexes (Zagwijn, 1985). A shift towards larger climate variation and more severe glacials is also 
evident in the Netherlands record at about $1 \mathrm{Ma}$ after the Menapian Stage. Sejrup et al. (1987) provide evidence for extensive glaciation of the central and northern North Sea in the later parts of the Matuyama, probably after about $0.9 \mathrm{Ma}$, which agrees with the evidence for large glacials after $1.2 \mathrm{Ma}$ in the record from Hole 644A (Fig. 6).

Some of the evidence from the Arctic and sub-arctic regions may contrast with the results from Leg 104. Glacial advances on Iceland have been dated as old as $3 \mathrm{Ma}$ (Einarsson et al., 1967), before the first major evidence from IRD in the Leg 104 sites indicating glacial expansion to the Scandinavian coastline. Reports from studies of deep-ocean cores from the central Arctic Ocean indicated glacial environments as early as the late Miocene (Clark, 1982). Jones (1987) reevaluated the magnetic time scale used in the Arctic Ocean studies and concluded that none of the cores contain sediments older than $2.5 \mathrm{Ma}$. If so, a discrepancy might not exist between the Arctic Ocean and Norwegian Sea records concerning the onset of large glaciations. Alternatively, there might be significant difference in the timing of glacial onset between the Arctic and the areas on the eastern side of the Norwegian Sea. Uplifted marine deposits from Alaska (Carter et al., 1986) and from Kap København (northern tip of Greenland) (Funder et al., 1985) document environments indicating ice-free conditions in the Arctic and a much more northerly position of the forest/tundra boundary than today. Paleomagnetic measurements indicate that these environments persisted during a period of a reversed magnetic polarity, and the authors suggest that these ice-free conditions are of Matuyama age. The oceanic heat needed to keep the Arctic Ocean ice free and to warm northern Greenland probably would pass through the Norwegian Sea in a manner similar to the present northward heat flux provided by the Norwegian Current. We have found no indications of periods with particularly warm climates in the Matuyama, however. On the contrary, even the warmer periods of the Matuyama appear significantly colder than the Holocene. The sample spacing used in this and other studies presented in this volume should be dense enough to identify extensive warm intervals as shown in the sections from northern Greenland. The evidence of a warm and forested Arctic is much easier to reconcile with the environmental conditions inferred from of the Norwegian Sea prior to the glacial onset at $2.56 \mathrm{Ma}$.

\section{PALEOENVIRONMENTS 0.73-0 Ma}

The Brunhes Epoch and the Matuyama were dissimilar in many respects. The earlier Brunhes marks the final transition into the climatic and oceanographic state that is typified by previous studies of upper Quaternary sediments in the NorwegianGreenland Sea. Interglacials were short, but realatively warm. Sedimentation was characterized by carbonate deposition, and ice-free conditions permitted deep-water formation as in the present ocean. The planktonic foraminifer data of Spiegler and Jensen, (this volume) show however, that only a few of these interglacials reached a warmth comparable to the Holocene. Henrich's (this volume) data on calcite dissolution also shows that dissolution was more widespread in glacials during the earlier parts of the Brunhes than in the late Brunhes where dissolution is only prevalent during short deglacial periods. The isotopic and IRD records show prolonged glacial phases bracketed by short interglacials. The presence of planktonic foraminifers and absence of benthics through most of the Brunhes (in contrast with the Matuyama) is indicative of a general deepening of the calcite lysocline, but also of less favorable conditions for benthic foraminifer productivity probably caused by rapid deposition rates of terrigenous, glacial muds.

Although some of the structure in the planktonic oxygen isotopic records can be attributed to low-salinity excursions, the Brunhes record appears less noisy than that of the transitional period in the late Matuyama. The glacial-to-interglacial $\delta^{18} \mathrm{O}$ amplitude of 1.5 to $2.0 \%$ is comparable with other upper Quaternary records (Kellogg et al., 1978; Jansen and Erlenkeuser, 1985; Beyer, 1988). The benthic isotope record of the Brunhes is very scattered, and only a few measurements indicate true interglacials. Despite this lack of resolution, the absolute values support the results from high-resolution studies of the late Quaternary Norwegian Sea, that glacial-to-interglacial contrasts in benthic oxygen isotopes were low, probably about $1.1 \% 0$ (Labeyrie et al., 1987).

\section{CONCLUSIONS}

1. A major expansion of the Scandinavian ice sheet to coastal areas occurred at approximately $2.6 \mathrm{Ma}$ and marked the initiation of repetitive glacial cycles in the Norwegian Sea. Relatively high-amplitude glacials appeared until about $1.9 \mathrm{Ma}$. The period 1.9 to $1.2 \mathrm{Ma}$ was characterized by relatively small icesheet fluctuations both in Scandinavia and globally. Starting at 1.2 Ma, ice-sheet variations increased markedly in amplitude, as indicated by larger input of IRD and oxygen isotopes, and the largest amplitudes occurred in the last $0.6 \mathrm{Ma}$. The shift toward higher amplitude climatic and oceanic fluctuations around 0.9 $\mathrm{Ma}$ appeared to be gradual and lasted for some hundred thousand yr.

2. Most of the Matuyama Chron (until approximately 0.9 $\mathrm{Ma}$ ) was characterized by weak inflow of warm surface waters and a cool climate, with markedly less climatic amplitudes than in the Brunhes. Interglacial stages were colder than the Holocene and a strong thermal gradient was present between the Norwegian Sea and the North Atlantic, in contrast to the present situation. These findings indicate a more zonal and less meridional climate system in the North Atlantic region than at present.

3. A persistant calcite dissolution period characterized most of the Matuyama until about $1 \mathrm{Ma}$. Together with carbon isotope evidence, this indicates deep waters enriched in $\mathrm{CO}_{2}$ and reduced deep-water ventilation for extended periods during the studied interval. Oxygen isotopic evidence suggests colder deep waters in the Norwegian Sea than in the North Atlantic during the studied period and also during the dissolution interval, thereby indicating deep-water exports to the North Atlantic in the Matuyama. This deep water apparently formed by other mechanisms than that forming under present conditions.

4. Although the study shows repeated glacial fluctuations during the last $2.6 \mathrm{Ma}$, the mode of variation has shifted, and it was only during the last $0.6 \mathrm{Ma}$ that the oceanic and climatic system of the Norwegian Sea fluctuated in the manner described by previous studies of Quaternary sediments from this region.

\section{ACKNOWLEDGMENTS}

We thank Captain Oonk, the crew of the JOIDES Resolution, and the shipboard technical and scientific party for shipboard assistance. The East Coast Core Repository and ODP is thanked for providing us with samples from Leg 94. R. Aspenes Justad, K. Nesbø, K.-J. Karlsen, S. Jakobsen, and P. Vallevik helped with sample processing in Bergen. We thank D. Hoddell and L. Labeyrie for careful reviews and many helpful suggestions which improved the manuscript. This study was supported by grants from the Norwegian Research Foundation for Science and the Humanities (NAVF-grants D.49.-31.002, D.41.31.133, and D.41.31.151) and the German Research Foundation (DFG-grant Th200314 and grants for the Joint Research Project SFB 313). A visiting scientist grant to the first author, from SFB 313 at Kiel University (DFG), is greatfully acknowledged. This report is a publication from the Joint Research Project (SFB) 313 in Kiel, FRG.

\section{REFERENCES}

Aagaard, K., Swift, J. H,. and Carmack, E. C., 1985. Thermohaline circulation in the Arctic Mediterranean Seas. J. Geophys. Res., 90: 4833-4846. 
Beyer, I., 1988. Sen-Kvarter paleoseanografi i det sentrale Islandshav. [Unpubl. Cand. Sci. thesis]. University of Bergen, Norway.

Carter, L. D., Brigham-Grette, J., Marincovitch, L., Pease, V. L., and Hillhouse, J. W., 1986. Late Cenozoic Arctic Ocean sea ice and terrestrial paleoclimate. Geology, 14:675-678.

Clement, B. D. and Robinson, F., 1987. The magnetostratigraphy of Leg 94 sediments. Init. Repts. DSDP, 94:635-650.

Clark, D. L., 1982. Origin, nature and world climate effect of Arctic Ocean ice cover. Nature, 300:321-325.

Duplessy, J. C., 1982. Circulation des eaux profondes Nord Atlantiques au cours du dernier cycle climatique. Bull. Inst. Geol. Bassin Aquitaine, 31:379-391.

Duplessy, J. C., Moyes, J. and Pujol, C., 1980. Deep water formation in the North Atlantic Ocean during the last ice age. Nature, 286:479482.

Einarsson, T., Hopkins, D. M., and Doell, R. R., 1967. The stratigraphy of Tjornes, northern Iceland, and the history of the Bering Land Bridge. In Hopkins, D. M. (Ed.), The Bering Land Bridge: Stanford, CA (Stanford University Press), 312-315.

Eldholm, O., Thiede, J., Taylor, E., et al. (1987). Proc. ODP, Init. Repts., 104: College Station, TX (Ocean Drilling Program).

Funder, S., Abrahamsen, N., Bennike, O., and Feyling-Hanssen, R. W., 1985. Forested Arctic: Evidence from North Greenland. Geology, 13:542-546.

Graham, D. W., Corliss, B. H., Bender, M. L., and Keigwin, L. D., 1981. Carbon and oxygen disequilibria of recent deep-sea benthic foraminifera. Mar. Micropaleontol., 6:483-497.

Grose, W. L., and Hoskins, B. J., 1979. On the influence of orography on large scale atmospheric flow. J. Atmos. Sci., 36:223.

Henrich, R., 1986. A calcite dissolution pulse in the Norwegian-Greenland Sea during the last deglaciation. Geol. Rundsch., 75:805-827.

Jansen, E. and Erlenkeuser, H., 1985. Ocean circulation in the Norwegian Sea during the last deglaciation: isotopic evidence. Palaeogeogr., Palaeoclimatol., Palaeoecol., 49:189-206.

Jansen, E. and Sejrup, H. P., 1987. Stable isotope stratigraphy and amino-acid epimerization for the last $2.4 \mathrm{~m} . y$. at Site 610, holes 610 and 610A. Init. Repts. DSDP, 94, 879-887.

Johannessen, T., 1987. Resente planktoniske foraminiferer fra Norskehavet, Islandshavet og Nord-Atlanteren: Taksonomi, faunafordeling og stabil-isotopsammensetning. [Unpubl. Cand. Sci. thesis]. University of Bergen, Norway.

Jones, G., 1987. The central Arctic Ocean sediment record: Current progress in moving from a litho- to a chronostratigraphy. In Symposium on late Cenozoic paleoenvironments and geology of the Arctic: Spidsbergseter (Norwegian Polar Research Institute). (Abstract).

Keigwin, L. D., 1987. Pliocene stable isotope record of Deep Sea Drilling Project Site 606: sequential events of ${ }^{18} \mathrm{O}$ enrichment beginning at 3.1 Ma. Init. Repts. DSDP., 94:911-920.

Kellogg, T. B., 1976. Late Quaternary climatic changes: evidence from deep-sea cores of Norwegian and Greenland Seas. In Cline, R. M., and Hays, J. D., (Eds.), Investigation of Late Quaternary Paleoceanography and Paleoclimatology. Geol. Soc. Am. Mem., 145:77-110.

Kellogg, T. B., Duplessy, J. C., and Shackleton, N. J., 1978. Planktonic foraminiferal and oxygen isotopic stratigraphy and paleoclimatology of Norwegian Sea deep-sea cores. Boreas, 7:61-73.

Labeyrie, L. D., and Duplessy, J. C., 1985. Changes in the oceanic ${ }^{13} \mathrm{C} /$ ${ }^{12} \mathrm{C}$ ratio during the last 140,000 years: high-latitude surface water records. Palaeogeogr., Palaeoclimatol., Palaeoecol., 50:217-240.
Labeyrie, L. D., Duplessy, J. C., and Blanc, P. L., 1987. Variations in the mode of formation and temperature of oceanic deep waters over the past 125,000 years. Nature, 327:477-482.

Mackensen, A., Sejrup, H. P., and Jansen, E., 1985. The distribution of living benthic foraminifera on the continental slope and rise off southwest Norway. Mar. Micropaleontol., 9:275-306.

Manabe, S., and Terpstra, T. B., 1974. The effects of mountains on the general circulation of the atmosphere as identified by numerical experiments. J. Atmos. Sci., 31:3.

Mix, A. C., and Fairbanks, R. G., 1985. North Atlantic surface ocean control of Pleistocene deep-ocean circulation. Earth Planet. Sci. Lett., 73:231-243.

Prell, W. L., 1982. Oxygen and carbon stratigraphy for the Quaternary of Hole 502B: evidence for two modes of isotopic variability. Init. Repts. DSDP, 68:269-276.

Ruddiman, W. F., Shackleton, N. J., and McIntyre, A., 1986a. North Atlantic seas-surface temperatures for the last 1.1 million years. Geol. Soc. Spec. Publ. (London) 21:155-173.

Ruddiman, W. F., Raymo, M. and McIntyre, A., 1986b. Matuyama 41,000-year cycles: North Atlantic Ocean and Northern Hemisphere ice-sheets. Earth Planet. Sci. Lett., 80:117-129.

Sejrup, H. P., Aarseth, I., Ellingsen, K. L., Lovlie, R., Reither, E., Bent, A., Brigham-Grette, J., E., Larsen, E. and Stoker, M., 1987. Quaternary stratigraphy of the Fladen area, central North Sea: a multidisciplinary study. J. Quat. Sci., 2.

Shackleton, N. J., and Opdyke, N. D., 1973. Oxygen-isotope and paleomagnetic stratigraphy of equatorial Pacific core V28-239: oxygen isotope temperatures and ice-volumes on a $10^{5}$ and $10^{6}$ year scale. Quat. Res., 3:39-55.

Shackleton, N. J., Imbrie, J., and Hall, M. A., 1983. Oxygen and carbon isotope record of east Pacific core V19-30: implications for the formation of deep water in the North Atlantic. Earth Planet. Sci. Lett., 65:223-224.

Shackleton, N. J., and Hall, M. A., 1984. Oxygen and carbon isotope stratigraphy of Deep Sea Drilling Project Hole 552A: Plio-Pleistocene glacial history. Init. Repts. DSDP, 81:599-609.

Shackleton, N. J., Backman, J., Zimmerman, H., Kent, D. V., Hall, M. A., Roberts, D. G., Schnitker, D., Baldauf, J. G., Desprairies, A., Homrighausen, R., Huddlestun, P., Keene, J. B., Kaltenback, A. J., Krumsieck, K A.D., Morton, A. C., Murray, J. W., and Westeberg-Smith, J., 1984. Oxygen isotope calibration of the onset of ice-rafting and history of glaciation in the North Atlantic region. Nature, 307:620-623.

Woodruff, F., Savin, S. M., and Douglas, R. G., 1980. Biological fractionation of oxygen and carbon isotopies by Recent benthic foraminifera. Mar. Micropaleontol., 5:3-11.

Zagwijn, W. H., 1985. An outline of the Quaternary stratigraphy of the Netherlands. Geol. Mijnbouw, 57:577-588.

Zahn, R., 1986. Spatquartäre Entwicklung von Küstenauftrieb und Tiefwasserzirkulation im Nordost-Atlantik. Rekonstruktion anhand Isotopen kalkschaliger Foraminferen [Ph. D thesis]. University of Kiel, FRG.

Date of initial receipt: 21 July 1987

Date of acceptance: 4 April 1988 Ms 104B-183

Ms 104B-183 